\title{
Determining the Neurotransmitter Concentration Profile at Active Synapses
}

\author{
Annalisa Scimemi • Marco Beato
}

Received: 6 July 2009 /Accepted: 30 September 2009 /Published online: 22 October 2009

(C) The Author(s) 2009. This article is published with open access at Springerlink.com

\begin{abstract}
Establishing the temporal and concentration profiles of neurotransmitters during synaptic release is an essential step towards understanding the basic properties of inter-neuronal communication in the central nervous system. A variety of ingenious attempts has been made to gain insights into this process, but the general inaccessibility of central synapses, intrinsic limitations of the techniques used, and natural variety of different synaptic environments have hindered a comprehensive description of this fundamental phenomenon. Here, we describe a number of experimental and theoretical findings that has been instrumental for advancing our knowledge of various features of neurotransmitter release, as well as newly developed tools that could overcome some limits of traditional pharmacological approaches and bring new impetus to the description of the complex mechanisms of synaptic transmission.
\end{abstract}

Keywords Time course · Diffusion .

Competitive low-affinity antagonist - Neurotransmitter . Synapse

\footnotetext{
A. Scimemi $(\square)$

Synaptic Physiology Section,

Porter Neuroscience Research Center,

National Institute of Neurological Disorders and Stroke,

National Institutes of Health,

35 Convent Drive, Building 35, Room 3C-1006,

Bethesda, MD 20892-3701, USA

e-mail: scimemia@ninds.nih.gov

M. Beato $(\square)$

Department of Neuroscience, Physiology and Pharmacology,

University College London,

London WC1E 6BT, UK

e-mail: m.beato@ucl.ac.uk
}

\section{Introduction}

The time course of synaptic currents is one of the main factors determining how a single neuron integrates information coming from different inputs. The duration of postsynaptic currents can vary over two orders of magnitude depending on the kinetics of the postsynaptic receptors being activated. For example, excitatory glutamatergic currents decay with time constants $\left(\tau_{\text {decay }}\right)$ as short as $2 \mathrm{~ms}$ when mediated by AMPA receptors alone (AMPARs), while the recruitment of NMDA receptors (NMDARs) prolongs the decay up to hundreds of milliseconds. Similarly, glycine-receptor-mediated inhibitory currents are fast $\left(\tau_{\text {decay }} \sim 3 \mathrm{~ms}\right)$ while $\mathrm{GABA}_{\mathrm{A}}$ receptor $\left(\mathrm{GABA}_{\mathrm{A}} \mathrm{R}\right)$ mediated events are approximately three to four times slower. Since these receptors operate in conditions of nonequilibrium, small variations in the neurotransmitter profile can alter considerably the time course of synaptic currents. It is normally thought that, following release, neurotransmitters in the cleft reach a peak concentration that is in the millimolar range, and decays rapidly $\left(\tau_{\text {decay }}=0.1-1 \mathrm{~ms}\right)$, due to diffusion, re-uptake, binding to receptors/transporters, or enzymatic breakdown. However, at some synapses in the central nervous system, receptors are exposed to small and prolonged neurotransmitter transients [1-4]. This longer concentration profile gives rise to synaptic currents that decay slowly (depending on the kinetic properties of the receptors) and leads to an extended period of synaptic excitation or inhibition. Therefore, determining the concentration profile of neurotransmitters released during synaptic events is essential for understanding the effects of individual synaptic inputs onto their corresponding postsynaptic targets. The major difficulties to address this issue experimentally are posed by the inaccessibility of narrow synaptic clefts, uncertainties in 
the ultrastructure, and physical constraints to neurotransmitter diffusion within and outside the cleft, and by the fact that synapses vary widely in shape, size, density of neurotransmitter receptors, and quantal properties of neurotransmitter release [5-10]. As a consequence, estimates of the neurotransmitter time course can only be rough approximations, often representing average estimates obtained through indirect approaches, primarily pharmacological, which can only partially characterize a few aspects of this phenomenon.

Here, we discuss the various features of synaptic release that can lead to different neurotransmitter concentration profiles in the cleft. We describe the pitfalls and advantages of the experimental approaches used so far, and briefly overview some of the emerging imaging approaches that could refine current estimates of the neurotransmitter lifetime once liberated in the synaptic cleft.

\section{Quantal Size and Multivesicular Release}

At first sight, an estimate of the neurotransmitter concentration profile in the synaptic cleft could be easily obtained if one knew how many neurotransmitter molecules are stored in each synaptic vesicle, how many vesicles fuse with the presynaptic terminal in response to an action potential, and how much of the vesicle content is released in the synaptic cleft with every partial or full fusion event. In practice, getting detailed information about each one of these steps has proven to be a challenging task.

The first attempt to measure how much neurotransmitter is released at the neuromuscular junction was probably done by Acheson [11], who suggested that $10^{-15}$ mmoles acetylcholine $(\mathrm{ACh})$ is released per presynaptic nerve stimulation. This value was inferred from titration experiments through exogenous ACh applications which, however, reproduced only roughly the time course of miniature end-plate potentials (mEPPs). Iontophoresis approaches proposed a tenfold increase in the above value $\left(10^{-14}\right.$ mmoles [12]), but still suffered from profound underestimates of ACh dilution from the iontophoresis pipette to the end-plate and presumably from overestimates of the end-plate area where the ACh receptors (AChRs) are distributed (the fraction of the total iontophoretic current passed by $\mathrm{ACh}$ was also an approximation [13]). The major breakthroughs came from two studies that provided information on: (1) the postsynaptic area activated by a quantum of neurotransmitter $\left(<2 \mu \mathrm{m}^{2}[14]\right)$; (2) the number of $\mathrm{ACh}$ molecules released by each quantum [13]. It is worth noting that for the experiments described by [13], the snake end-plate preparation was placed in an oil-water interface, which therefore minimized the dilution artifacts mentioned previously. The authors performed two consecutive titrations to calculate the charge that had to be applied to the iontophoresis pipette to reproduce a mEPP reasonably accurately, and the number of ACh molecules released from the iontophoresis pipette per unit of charge. The final approximation of $<10^{4}$ ACh molecules/vesicle suggested that in a postsynaptic area of $1 \mu \mathrm{m}^{2}$, with a 50-nm-wide synaptic cleft, the peak ACh concentration is $\sim 0.3 \mathrm{mM}$, and in a 50-nm-wide synaptic vesicle the ACh concentration is $\sim 260 \mathrm{mM}$ [13]. There is rough agreement between these estimates and the more detailed ones obtained later on at the frog and mouse neuromuscular junctions (peak neurotransmitter concentration in the cleft $\sim 1 \mathrm{mM}$, but see Fig. 2 in [15] for an evaluation of how the ACh concentration changes as it spreads over the postjunctional area). Therefore, to this day, the hypothesis that synaptically released $\mathrm{ACh}$ reaches millimolar concentrations at the neuromuscular junction remains the most widely accepted.

The use of carbon-fiber microelectrodes and amperometry has been instrumental to obtaining analogous estimates from cells that release electro-oxidizable molecules such as catecholamines and indoleamines. Although the technique was initially developed to detect neurotransmitter release from chromaffin cells [16], it has also been used on central neurons [17]. Briefly, electro-active neurotransmitters are oxidized by the carbon-fiber, and the reaction causes a positive deflection in the current measurement obtained through the microelectrode. A read-out of the vesicular neurotransmitter concentration is readily obtained by converting the charge transfer during the positive deflection into number of moles by using Faraday's constant. Accordingly,

$N=\frac{Q}{n \cdot F}$

where $N=$ number of moles of neurotransmitter, $Q=$ charge transfer per positive deflection (C), $n=$ number of electrons involved in the reaction, $F=$ Faraday constant. It was estimated that the vesicular concentration of catecholamine in chromaffin cells is $190-300 \mathrm{mM} \mathrm{[16]} \mathrm{and} \mathrm{that} \mathrm{of}$ dopamine in midbrain neurons is $\sim 300 \mathrm{mM}$ [17].

Amperometric measures offer excellent time resolution, but because neurotransmitters are degraded as they react with the carbon-fiber, estimates of the time course of release can potentially be slightly inaccurate (i.e., the neurotransmitter concentration decreases as it reacts with the carbonfiber). The major limitations of this technique, however, are posed by the facts that carbon-fibers (which have a diameter of a few micrometers) cannot access the synaptic cleft (which has a width of tens of nanometers), and are not suited for neurotransmitter concentration measurements at synapses that do not release non electro-oxidizable molecules.

At central synapses, direct measures of the vesicular concentration of glutamate (as well as GABA, glycine, and 
other neurotransmitters) have not been obtained. However, numerous biochemical studies have been crucial to provide some sort of ballpark figure. For example, immunoisolation of synaptic vesicles with antibodies against synaptophysin has shown that, under experimental conditions that enable the maintenance of an energy gradient across the vesicular membrane, glutamate is ten times more concentrated in synaptic vesicles that in the cytoplasm [18]. Given that (1) there is $\sim 0.8 \mu \mathrm{mol}$ glutamate/mg synaptophysin, (2) synaptophysin represents $\sim 7 \%$ of total vesicle proteins, (3) the volume of synaptic vesicles is assumed to be $\sim 1 \mu \mathrm{l} / \mathrm{mg}$ total protein, it was suggested that the glutamate concentration in synaptic vesicles is $\sim 60 \mathrm{mM}$, and certainly $<150 \mathrm{mM}$, a value imposed by the Donnan law [18]. These estimates are in rough agreement with those obtained from synaptic vesicles isolated with other purification protocols, yielding values of 70-210 $\mathrm{mM}$ [19].

It is worth noting, however, that all these quantifications have been obtained through a series of conversions that at some point require knowledge of the inner volume of synaptic vesicles. This information has been traditionally obtained through electron microscopy studies, but the size of synaptic vesicles can be altered by the chemical fixation steps required for tissue preparation (see [20] for one of the most detailed analyses of synaptic vesicle structure). Since the synaptic vesicle volume scales with the third power of its radius, small errors in length can lead to much bigger errors in volumes. Therefore, it is still unclear whether the synaptic vesicle volume varies significantly across the entire population of synaptic vesicles in presynaptic terminals [20, 21] or not [22]. There is also some debate on whether the neurotransmitter concentration varies across synaptic vesicles of different size [23, 24], or with different copies of vesicular neurotransmitter transporters [25]. Since the final neurotransmitter concentration in the cleft depends on the total number of neurotransmitter molecules released, vesicles with similar neurotransmitter concentration but different volume can still lead to different neurotransmitter concentration profiles in the cleft.

Do synapses always release one vesicle or are they also capable of releasing more than one? Despite the fact that multiple vesicles are docked at most presynaptic terminals, the number of active synaptic connections among neurons generally exceeds the total number of quanta released during synaptic stimulations [26, 27]. This combined anatomical and electrophysiological evidence has led to the hypothesis that, at various peripheral and central synapses, not more than one vesicle of neurotransmitter is released from a given active zone (univesicular release hypothesis) [28, 29]. If the hypothesis holds for those single-site connections where quantal events can be clearly resolved (i.e., when miniature events are much bigger than the baseline noise of the recordings, and are not distorted by the cable properties of neuronal dendrites [30]), it does not necessarily imply a unique mode of release in time and across the different synaptic contacts that a neuron forms [31].

In the last decade, numerous studies have proposed that multiple release events can also occur (multivesicular release hypothesis) [30, 32-41]. One line of evidence in support of multivesicular release is the occurrence of miniature currents that arise in brief succession, do not summate linearly, and are not independent on one other [30]. A more indirect approach relies on the measure of the amplitude of evoked EPSCs when varying release probability $\left(P_{\mathrm{R}}\right)$ in the presence of low-affinity competitive antagonists [32, 34, 40] (described in a following section of this review). Such antagonists are less efficient in blocking the response when the receptors are exposed to higher concentrations of neurotransmitter. Consequently, if increasing $P_{\mathrm{R}}$ increases the amount of neurotransmitter released from individual synapses (as suggested by the multivesicular release hypothesis), it also leads to reduced sensitivity of postsynaptic currents to low-affinity antagonists. Conversely, if increasing $P_{\mathrm{R}}$ only increases the number of active synapses, but each synapse still releases one quantum, no change in the effect of low-affinity antagonists on postsynaptic currents is expected [32, 34, 40]. Multivesicular release can result in a prolonged exposure time of postsynaptic receptors to the agonist if the neurotransmitter vesicles are not released simultaneously, thereby leading to postsynaptic currents that decay more slowly. This, however, is a rather unspecific effect, that can arise for other reasons than multivesicular release. For example, it can occur when varying the mode of vesicle fusion [42] or the extent of neurotransmitter spillover among neighboring synapses [43]. A clear distinction between these scenarios is difficult to establish and it is probably for this reason that it remains arduous to unequivocally prove the occurrence of multivesicular release at certain synapses.

The extent to which the neurotransmitter concentration profile in the cleft is altered by multivesicular release also depends on the temporal characteristics of each synaptic vesicle fusion, and on the number of neurotransmitter molecules released during successive fusion events. For example, if only a few neurotransmitter molecules were released in the cleft during the second fusion, the profile of the neurotransmitter transient would be only marginally different from that observed when univesicular release takes place.

From a functional standpoint, having the ability to release more than one synaptic vesicle endows synapses with the ability to expand their dynamic range of outputs in response to synaptic stimulations and presumably to enhance the reliability with which information is conveyed from one neuron to the other. This simple scenario, however, may be complicated by the fact that the magnitude of the postsynaptic response and the pattern of activity of the postsynaptic neuron can be 
shaped by non-linear interactions between neurotransmitter receptors and voltage-gated conductances [41].

\section{Partial Versus Full Fusion Events}

$\mathrm{Ca}^{2+}$ entry in the presynaptic terminal triggers a complex cascade of events that ultimately results in fusion of neurotransmitter vesicles with the presynaptic membrane. Whether this process involves complete or transient fusion between vesicles and synaptic terminals remains a hotly debated issue that began with the ultrastructural detection of omega-shaped profiles at the active zone of the neuromuscular junction after prolonged electrical stimulation [44, 45]. Because electron microscopy only provides a snapshot of an ongoing process, however, it remained unclear whether these structures represented a vesicle in the process of releasing its content [45] or one that was being immediately retrieved [44]. Since these initial observations a number of functional approaches ranging from amperometry [46] to capacitance measurements [47] and imaging of fluorescent styryl dyes and $\mathrm{pH}$ sensors [48, 49] have confirmed the occurrence of transient fusion although, to date, it remains unclear whether this represents a typical or rare mode of neurotransmitter release.

At some synapses, the net prevalence of partial/full fusion events can be tested by performing capacitance measurements from the presynaptic terminal [47]. Since membrane capacitance is linearly proportional to cell surface, it increases any time a vesicle fuses with the presynaptic membrane. Accordingly, step-like increases and decreases in cell capacitance have been observed in chromaffin cells and neurons $[47,50,51]$. These are attributed to vesicle fusion and retrieval events, respectively, because (1) the amplitude distribution of capacitance steps matches that of synaptic vesicle surface area obtained through morphological studies [51, 52]; (2) the time course of fluorescence loss from dye-loaded synaptic vesicles matches that of cell capacitance increase [52]; (3) the frequency of the up-steps, like that of release events, depends on the external $\left[\mathrm{Ca}^{2+}\right]$ and drops in the presence of the protease trypsin [47]; (4) up-steps can only be recorded from the side of presynaptic terminals that faces the postsynaptic cell [47].

The capacitance measurements are generally performed by coupling a phase lock-in amplifier with a voltage-clamp amplifier. The cell (or the patch, if the recordings are in cell-attached configuration) is voltage-clamped and forced to undergo sinusoidal voltage membrane oscillations. The lock-in amplifier decomposes the real (Re) and imaginary components (Im) of the admittance, which are in-phase and $90^{\circ}$-out-of-phase with the membrane voltage, respectively. Changes in Re reflect conductance changes, whereas changes in Im reflect changes in capacitance. Therefore, one can also obtain information on the fusion pore conductance $\left(G_{\mathrm{P}}\right)$ by applying the equation:

$G_{\mathrm{P}}=\frac{\mathrm{Re}^{2}+\mathrm{Im}^{2}}{\operatorname{Re}}$

whereas the diameter of the fusion pore $\left(D_{\mathrm{P}}\right)$ is expressed as:

$D_{\mathrm{P}}=\sqrt{\frac{4 G_{\mathrm{P}} \rho \lambda}{\pi}}$

( $\rho=$ saline resistivity $(100 \Omega \mathrm{cm}), \lambda=$ pore length (generally set at $15 \mathrm{~nm}$, the length of a gap junction hemichannel)) [53]. The time constant of neurotransmitter discharge can be described as:

$\tau=\frac{\pi d_{\mathrm{V}}^{3}}{6 \rho D^{*} G_{\mathrm{P}}}$

$\left(d_{\mathrm{V}}=\right.$ vesicle diameter, $D^{*}=$ apparent neurotransmitter diffusion constant).

At the calyx of Held, $97 \%$ of the events detected with capacitance measurements have $G_{\mathrm{P}}>288 \mathrm{pS}$ and $D_{\mathrm{P}}>2.3 \mathrm{~nm}$, and are interpreted as full fusion events because they can lead to complete neurotransmitter release from synaptic vesicles in $\tau<0.54 \mathrm{~ms}$. If this were true, miniature excitatory postsynaptic currents (mEPSCs) would be predicted to rise with $10-90 \%$ rise time $=0.15-0.36 \mathrm{~ms}$, a range that closely matches the one observed experimentally for most mEPSCs $(0.21-0.27 \mathrm{~ms})$ [47]. For the remaining $3 \%$ of the events $G_{\mathrm{P}}=66 \mathrm{pS}$, leading to $D_{\mathrm{P}}=1.1 \mathrm{~nm}$ and $\tau=2.3 \mathrm{~ms}$ [47]. In this case, the probability of releasing completely the synaptic vesicle content in the extracellular space (ECS) depends on how long the fusion pore remains open. This parameter varies across a relatively broad range (10 ms-1 s), so that the rare occurrence of nonquantal events cannot be completely ruled out.

At hippocampal synapses, partial fusion (although not necessarily partial neurotransmitter discharge) initially emerged as the predominant mode of neurotransmitter release $[54,55]$. This feature has been proposed to speed the recycling time of synaptic vesicles substantially, to 12 s [54] (cf. 15-20 s for clathrin-mediated endocytotic pathways $[56,57])$, and therefore enable reliable synaptic transmission during high-frequency stimulation. Such a fast recycling time would be primarily limited by the time of vesicular reacidification via the $\mathrm{H}^{+}$-pump and of neurotransmitter refilling through vesicular transporters. It should be noted, however, that this net prevalence of partial fusion events is not unanimously observed [57, 58] and may be biased by some methodological and analytical caveats [57]. For example: (1) lack of complete FM1-43 loss from synaptic vesicles may reflect dye partitioning in membranes neighboring the site of neurotransmitter release, rather than the occurrence of partial fusion events; (2) the fast decline in the fluorescence of the $\mathrm{pH}$-sensitive GFP synapto- 
pHluorin after exocytosis may be due to diffusion of the indicator away from the release site rather than to rapid endocytosis, and can be accounted for by enlarging the region of interest used for quantifying fluorescent signals [57]. The incidence of partial fusion events may vary across synapses with different release probabilities or experiencing different levels of synaptic stimulation [49], and possibly changes during development [59], but additional work will help to test these hypotheses in further detail [57].

One intriguing approach that has been recently used to measure the likelihood of partial/full fusion events involves the use of quantum dots as reporters of different vesicular $\mathrm{pH}$ and fusion modes [49, 60]. Quantum dots with a hydrodynamic diameter of $\sim 15 \mathrm{~nm}$ (matching the intravesicular diameter) have a peak emission at $605 \mathrm{~nm}$, with an intensity that apparently increases with $\mathrm{pH}$ [49]. The acidic $\mathrm{pH}$ of synaptic vesicles (5.5) rises to extracellular values (7.4) upon opening of the fusion pore. The consequent increase in the fluorescence signal from the quantum dots is recovered if the fusion pore closes back (partial fusion), but is followed by an irreversible loss of fluorescence if the quantum dot is released into the extracellular environment (full fusion) [49]. This approach suggests that partial fusion is limited to $\sim 20 \%$ of all fusion events, and involves primarily synaptic vesicles with relatively high intrinsic release probability (readily releasable pool [49]). A prevalence of full fusion events would shift the source of variability in the peak concentration of released neurotransmitter to differences in vesicle size and vesicle filling that we mentioned in the previous chapter [25].

In addition to the mode of release, the lifetime of the neurotransmitter in the ECS is also affected by a number of other factors. Once released into the synaptic cleft, the neurotransmitter is subject to three main phenomena: (1) binding to postsynaptic receptors or to degrading enzymes in the ECS; (2) diffusion and escape from the synapse; (3) binding to nearby transporter molecules. All these processes act in a concerted, but not necessarily simultaneous, way to rapidly terminate the neurotransmitter actions and enable reliable high-frequency signaling among neurons. In the next sections, we analyze how each one of them contributes to shape the lifetime of neurotransmitters in the synaptic cleft.

\section{Neurotransmitter Enzymatic Breakdown and Receptor Binding}

At the neuromuscular junction, $\mathrm{mEPPs}$ rise in $<1 \mathrm{~ms}$ and decay with a half-time of about $1-1.5 \mathrm{~ms}$ at room temperature. The duration and variability of mEPPs is enhanced in the presence of molecules like prostigmine or eserine, which inhibit the activity of ACh esterase, the degrading enzyme for ACh present in the ECS [61-63].
Hydrolysis of ACh is generally thought to be rapid and, based on the previous observations, to represent the major mechanism responsible for the fast time course of mEPPs at the neuromuscular junction [62]. When the enzymatic breakdown of $\mathrm{ACh}$ is reduced, however, the decay of mEPPs (3.5-4 ms) is not entirely accounted for by free diffusion of the neurotransmitter away from the cleft $(2 \mathrm{~ms}$ [64]), estimated through a simple analytic approach initially described by [65] and [66]. What other mechanism could underlie this discrepancy? Although one would have been naturally inclined to dismiss the accuracy of the adopted mathematical model, Katz and Miledi [62] perceptively noticed that the effects of ACh esterase inhibitors on the decay of mEPPs could be partially counteracted by curare or $\alpha$-bungarotoxin, antagonists of the nicotinic AChRs mediating the mEPPs. These drugs, per se, reduced the amplitude of the mEPPs, as well as their variability and decay time. The proposed hypothesis was that ACh unbinding and rebinding to its receptors delays the neurotransmitter clearance from the synaptic cleft. If one assumes that there are more AChRs than ACh molecules released during synaptic events, and that curare unbinding from AChRs is negligible during the time course of the mEPPs, then the duration of the ACh transient becomes inversely proportional to the number and lifetime of $\mathrm{ACh}-\mathrm{AChRs}$ complexes. That is to say: if $p$ is the fraction of ACh molecules initially bound to AChRs, the decay of the ACh transient is $1 /(1-p)$ slower than what it would be in the case of free diffusion $(p=0)$. This delay, as well as the variability of mEPPs amplitude, is largely reduced by curare because by lowering $p$ the drug brings the time course of cholinergic events close to the lifetime of single ACh-AChRs complexes.

The proposed mechanism strongly depends on the kinetic profile of the AChR-antagonist interaction and consequently may not be equally relevant for other pharmacological compounds. More importantly, it is unlikely to have a major impact when the density of postsynaptic receptors is low (like at central synapses and, possibly, at the neuromuscular junction itself). Although ACh is broken down extracellularly, apart from ATP and peptides, other transmitters are not degraded. Rather, they are subject to diffusion and reuptake, which therefore represent the main phenomena that shape the time course of synaptic events.

\section{Diffusion and Reuptake}

The time course of the concentration change of a solute in a solution is quantitatively described by Fick's second law of diffusion:

$\frac{\partial C_{x}}{\partial t}=D \frac{\partial^{2} C_{x}}{\partial X^{2}}$ 
where $C_{x}=$ concentration of the solute at distance $x$ from a reference point, $D=$ diffusion coefficient of the solute in free, aqueous solution, and $t=$ time. This formalism, which describes the temporal development of a concentration profile in a homogeneous and free medium, cannot be used as it is to define the movement of neurotransmitter molecules in the brain. The brain may not necessarily behave as a homogeneous (or isotropic) medium (although this may be a more or less of a concern for different brain regions); the apparent diffusion coefficient of the neurotransmitter in the ECS $\left(D^{*}\right)$ is generally smaller than the one measured in free solution $(D)$, due to the geometrical and molecular complexity of the ECS in respect to free medium [67]; part of the neurotransmitter molecules can be removed from the ECS as they are enzymatically degraded or taken up by transporters. An evaluation of the applicability of Fick's second law to the brain environment has been proposed by Nicholson and Phillips [67], by approximating this structure to a porous medium with extracellular volume fraction $\alpha$ and tortuosity $\lambda$. The equation, which now reads:

$\frac{\partial C}{\partial t}=\frac{D^{*}}{\lambda^{2}} \nabla^{2} C+\frac{Q}{\alpha}-v \times \nabla C-\frac{f(C)}{\alpha}$

takes into account the contribution of hindered diffusion (first term), the effective volume within which molecules move (second term), bulk flow (if any, third term) and neurotransmitter loss due to enzymatic breakdown, uptake, etc. (fourth term) $(C=$ neurotransmitter concentration, $t=$ time, $D^{*}=$ apparent neurotransmitter diffusion coefficient, $\lambda=$ tortuosity, $Q=$ source strength, $\alpha=$ extracellular volume fraction, $v=$ velocity vector) $[67,68]$. The variable $\alpha$ can be thought of as the fraction of brain tissue that is not occupied by neuronal or glial processes or by vasculature and that in electron micrographs may appear as a series of "empty" spaces (note that in reality the ECS is far from being "empty", hosting a variety of macromolecules and viscous components that contribute to the smaller value of $D^{*}$ in respect to $D$ ). Various methods indicate that in the brain of newborn mammals, on average, $\alpha=0.36-0.46$ and reaches a value of 0.2 during adulthood [69]. The tortuosity $\lambda$, instead, measures the extent to which diffusion in the brain is slowed down in comparison to free solution $\left(\lambda=\sqrt{D / D^{*}}\right)$. It does not change significantly with development $(\lambda \sim 1.6)$, but may vary across different brain regions and in different animal species $(1.39<\lambda<1.77$ [69] -note that this is a relatively wide range because it implies a $40 \%$ difference in $D^{*}$, which can influence significantly the extent of postsynaptic receptor activation [70]). Multiple factors contribute to $\lambda$ : the geometry of the ECS (e.g. number and shape of neighboring structures), dead-end microdomains [71], the ECS viscosity due to the presence of proteins and various macromolecules within the extra- cellular matrix, as well as any interactions between neurotransmitters and receptors or charged molecules in the ECS [68]. The geometric and viscous components of $\lambda$ are often referred to as $\lambda_{\mathrm{g}}$ and $\lambda_{\mathrm{v}}$. In a simplified $3 \mathrm{D}$ environment where the ECS resembles a series of packed convex cells, one could consider $\lambda=\lambda_{g} \cdot \lambda_{v}$ (but see $[72,73]$ for a more detailed mathematical evaluation of how dead-end pores contribute to brain tortuosity). The numerical range across which $\lambda_{\mathrm{g}}$ can vary is described by the equation $[68,74]$ :

$\lambda_{\mathrm{g}}=\sqrt{\frac{3-\alpha}{2}}$

Therefore, for $0<\alpha<1$, one has $1<\lambda_{\mathrm{g}}<1.225$. The discrepancy between this upper limit for $\lambda_{\mathrm{g}}$ and the experimental value of $\lambda=1.6$ is likely due to $\lambda_{v}$, the specific/non-specific binding effects mentioned previously (whose effective contribution to $\lambda$, however, remains undetermined) and the greater morphological complexity of the brain.

A quantitative estimate of $\alpha$ and $\lambda$ is not necessarily trivial. Whereas various methods have been explored to estimate $D^{*}$ or $\lambda$ [75-78], a simultaneous measure of $\alpha$ and $\lambda$, including both the viscous and the geometrical component, has only been obtained initially with radiotracers [79] and later on with real-time iontophoresis [67]. The use of radiotracers has been hampered by the fact that they require long perfusion times and post-mortem processing of the tissue [69]. The iontophoresis technique involves monitoring the diffusion of small ( $\mathrm{MW}<100 \mathrm{Da}$ ), cell-impermeable ions (e.g., tetramethylammonium, tetraethylammonium, $\alpha$ naphthalene sulfonate) from an iontophoresis electrode to an ion-selective one, mounted at a fixed distance from each other (few hundreds of micrometers) [67]. The signal detected by the ion-selective electrode is displayed as a diffusion curve which is used to extract the values of $\alpha$ and $\lambda$ (in addition to $k^{\prime}$, a measure of neurotransmitter loss [67]). The main caveat of this approach, as pointed out by [75], is that it only provides information about diffusion of small molecules towards one point in the tissue without resolving possible spatial patterns of diffusion. This limitation can be partly overcome with integrative optical imaging approaches [75, 77]. By monitoring the diffusion profile of fluorescently labeled molecules with high molecular weight (e.g. Texas Red dextrans up to tens of kilodaltons [75] or quantum dots [77]), one can derive $D^{*}$ for smaller neurotransmitter molecules. The technique relies on a mathematical description of the image of the threedimensional diffusion of dyes on a two-dimensional plane. To avoid this step, which nevertheless approximates the experimental results reasonably well [77], and to gain an even better time resolution of diffusion, the time-space profile of small fluorescent molecules (e.g., Alexa Fluo 350) can be examined in a thin focal plane, like that of a 
two-photon laser scanning microscope [76, 78]. In all cases, it is assumed that dyes diffuse in the ECS with radial symmetry from a point source. The goodness of this approximation can be verified post-hoc by analyzing the goodness of a Gaussian fit of the diffusion profile of the dye, which generally holds.

An exhaustive comparison of how the estimate of the diffusion parameters vary with different experimental approaches is still missing. Although in principle the twophoton approach offers the best spatial and time resolution, it remains unclear whether this effectively improves the accuracy of the numerical estimates. The major caveat common to all approaches, in our opinion, is that they all provide bulk measures of diffusion over distances (tens or hundreds of micrometers) and times (milliseconds) that largely exceed the spatiotemporal scales of synapses (nanometers, microseconds). An estimate of $D^{*}$ glutamate from physiological experiments has been performed by Nielsen et al. [80] through an evaluation of the effect of dextran on the kinetics and amplitude of spillover-mediated currents at cerebellar mossy fiber-granule cell synapses $\left(D_{\text {glutamate }}^{*}=0.33 \pm 0.13 \mu \mathrm{m}^{2} / \mathrm{ms}\right.$; mean \pm S.E.M. $)$, but it is currently unknown whether this estimate can be considered reasonably accurate for other types of synapses.

One major point of uncertainty is whether any change in neurotransmitter diffusion occurs between the synaptic cleft and neighboring extrasynaptic regions. Given that the majority of the tortuosity factor is contributed by geometrical factors, and there is no geometric obstacle to diffusion in the synaptic cleft, it could be feasible that $D^{*}$ cleft $<<$ $D^{*}$ extrasynaptic areas [81].

Why is it relevant to have such a meticulous estimate of $D^{*}$ and $\lambda$ in the brain? Slowly diffusing neurotransmitters persist in the ECS for a relatively long time, and have higher chances of binding to receptors. Therefore, by altering the neurotransmitter concentration profile, varying $D^{*}$ can also alter the open probability of receptors, as long as these are far from saturation. Indeed, this prediction has been tested in a number of simulation environments for a range of $D^{*}=0.1-0.75 \mu \mathrm{m}^{2} / \mathrm{ms}[80,82,83]$ (here the upper value equals the diffusion coefficient of glutamine in free medium at $25^{\circ} \mathrm{C}$ [84]) and also experimentally [85]. For example, at hippocampal mossy fiber synapses on CA3 pyramidal cells, lowering $D^{*}{ }_{\text {glutamate }}$ by $50 \%$ enhances significantly activation of AMPAR, NMDAR, and kainate receptors [85]. Whether even smaller changes in $D^{*}$ can still lead to physiologically relevant effects on receptor activation and inter-neuronal signaling remains to be evaluated: varying $D^{*}$ alters the neurotransmitter concentration profile, but whether this leads to small or big changes in postsynaptic responses depends on the properties of the activated receptors. By using the kinetic models of four receptor types described in Fig. 1, we have analyzed how significant changes in receptor activation could occur with neurotransmitter transients of different amplitude and duration (Fig. 2). A wide range of uncertainties in the estimate of the neurotransmitter concentration profile can alter significantly the peak open probability of postsynaptic receptors. For example, the extent of AMPAR activation is particularly susceptible to changes in the profile of the neurotransmitter transient. One could then speculate that this may alter profoundly the temporal integration of synaptic inputs, given the high temporal precision with which certain synapses can operate [86].

The effects of changes in $D^{*}$ could be particularly pronounced for extrasynaptic receptors scattered around the postsynaptic density and in dendritic shafts [70]. For example, at glutamatergic synapses the occupancy of high-affinity slow-desensitizing NMDARs may be enhanced by increasing the overall tortuosity of the synaptic and non-synaptic milieu [83]. The physiological relevance of a differential activation of extrasynaptic receptors remains a matter of current investigation.

Needless to say, activation of these distant receptors is extremely susceptible to the presence, distribution, and relative abundance of transporters which can quickly buffer neurotransmitter molecules in hundreds of microseconds $[39,87,88]$ and slowly remove them from the ECS, at rates that depend on the membrane potential, temperature, and concentration of co-transported ions $\left(\sim 4-27 \mathrm{~s}^{-1}\right.$ for $V_{\mathrm{R}}=$ $-140-0 \mathrm{mV}$, at RT [89]). A quantitative analysis of glutamate transporter density has been performed by Lehre and Danbolt [90], and appears to vary among different brain regions, depending on the developmental stage of the preparation. For example, in adult rats, the glutamate transporter GLT-1 is more abundantly expressed in hippocampal CA1 stratum radiatum $\left(8,500 \mu^{-2}\right)$ than in cerebellar stratum moleculare $\left(740 \mu^{-2}\right)$, but it is barely detectable in newborn rats [91]. In agreement with this developmental trend, the time course of glutamate clearance derived from astrocytic recordings in rat hippocampal stratum radiatum also becomes faster between $\mathrm{P} 12-14$ and $>$ P60 [92]. The contribution of transporters to glutamate clearance is enhanced at physiological temperature (perhaps not surprisingly: the temperature dependence of neurotransmitter uptake $\left(Q_{10}=3\right)$ is steeper than that of diffusion $\left.\left(Q_{10}=1.3\right)\right)$ [43] and may be exacerbated during repetitive presynaptic stimulations [93].

The activity of glial glutamate transporters has also been analyzed in the context of activation of particular receptor subtypes that have inhomogeneous subcellular distributions, like metabotropic glutamate receptors [94] or NR2Bcontaining NMDARs [95]. However, an exhaustive characterization of the role of neuronal glutamate transporters is lacking. Perisynaptic EAAT4 has been shown to control activation of metabotropic receptors in Purkinje cells [96], 


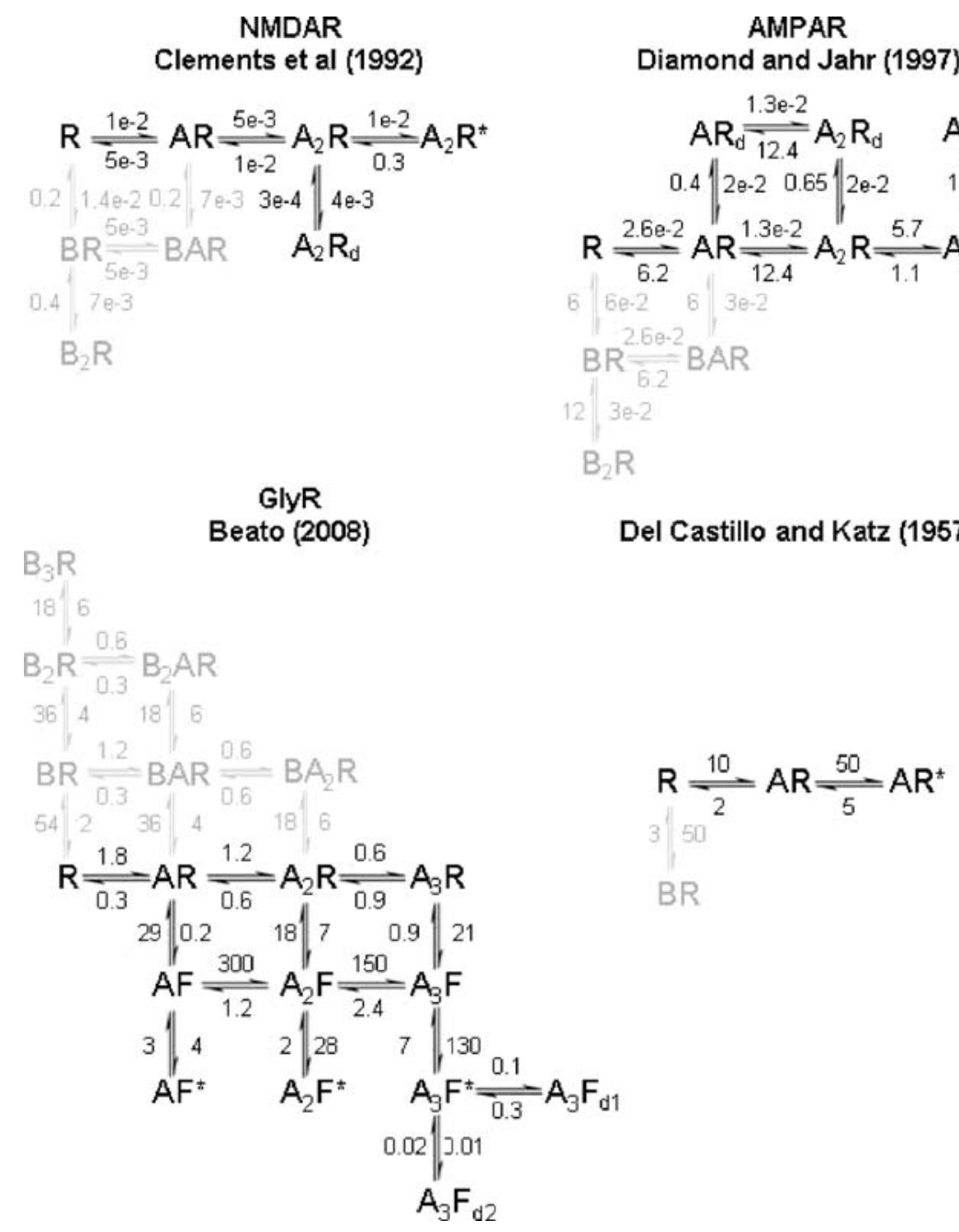

Fig. 1 The kinetic models used to estimate the time course of transmitter detected by NMDARs [105], AMPARs [88], glycine [107] receptors or for a generic agonist-receptor model [118]. All rates are taken from the original publications and are expressed in $\mathrm{ms}^{-1}$ or $\mathrm{mM}^{-1} \mathrm{~ms}^{-1}$, as appropriate. The rates for the model of del Castillo and Katz [118] are adapted to produce a current with a $\tau_{\text {decay }}=6 \mathrm{~ms}$. Agonist molecules are indicated by $A$, resting states of the receptor by $R$, and open states by an asterisk. $F$ indicates the flipped state of a

receptor, whereas the subscript $d$ identifies desensitized states. $B$ represents blocker molecules (e.g., low-affinity antagonists). Reactions in black have been used to simulate the synaptic responses in Fig. 2; reactions in gray represent binding of low-affinity antagonist blockers to their corresponding receptor molecules (D-AA, kynurenic acid and SR-95531 for NMDARs, AMPARs and glycine receptors, respectively). All reactions, in black and in gray, have been used for the analysis presented in Fig. 4

although not in regions lacking the protein zebrin II [97]. A definitive understanding of the role of EAAT3 is currently missing [98], partly because there is no sensitive antibody or specific pharmacological compound that can specifically target this molecule. Recent experimental and theoretical estimates suggest that EAAT3 could have a potentially pivotal role in regulating the profile of the glutamate transient $[78,98]$.

\section{Mathematical Simulations of Synaptic Currents}

The first attempts to calculate the time course of neurotransmitter release in the peripheral system were performed at a time when anatomical details about the shape and size of the synaptic cleft were still unavailable [99, 100]. These estimates, obtained by simply applying the formula for unhindered diffusion of a substance from a point source into free medium, were not necessarily aimed at providing a faithful representation of the neurotransmitter transient, yet they informed us on the ballpark time window over which diffusion of neurotransmitters could occur and triggered a long-lasting debate on the role of this phenomenon in shaping the profile of synaptic currents. A step forward was made in the late 1950s by Eccles and Jaeger [61], who performed a more explicit mathematical analysis of $\mathrm{ACh}$ diffusion in disk- or strip-like synaptic clefts where diffusion in the $z$-dimension was prevented by the presence 
NMDAR

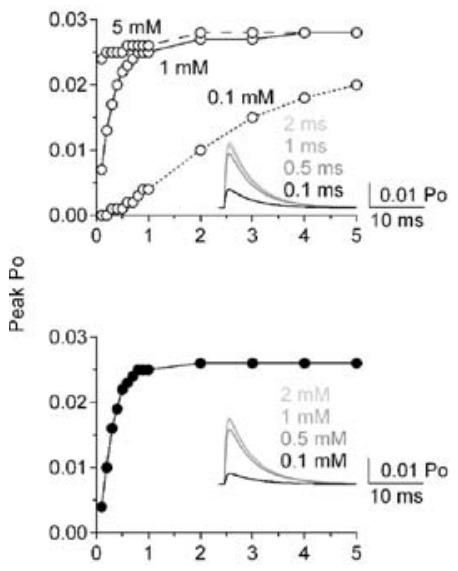

AMPAR

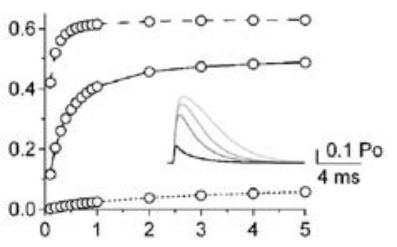

Duration of agonist pulse $(\mathrm{ms})$

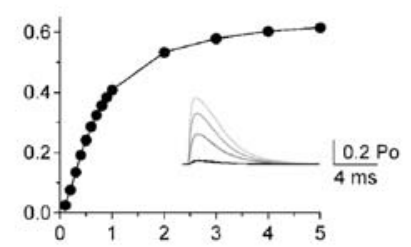

GlyR
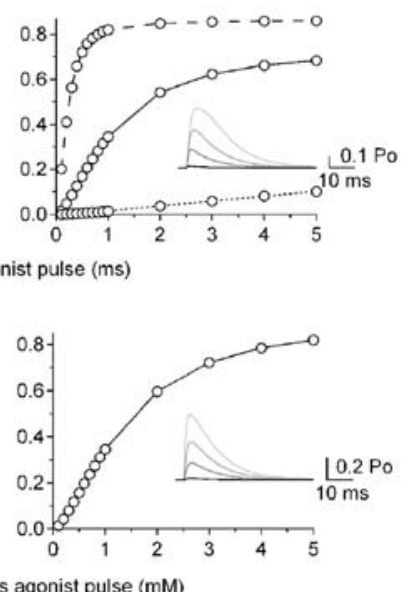

Del Castillo and Katz
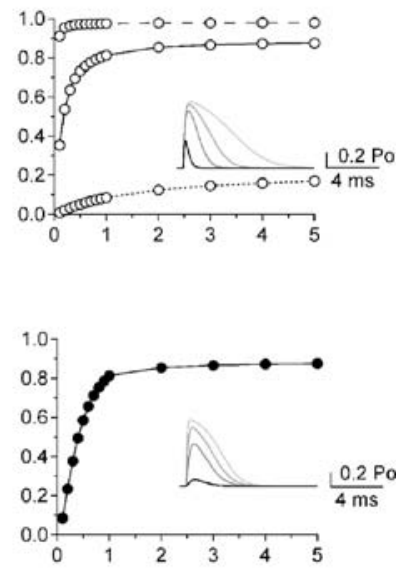

Fig. 2 Effect of altering the profile of the neurotransmitter transient on the occupancy of different receptors. We used a simplified version of the kinetic models described in Fig. 1, where the reactions between receptors and antagonists have been removed (i.e., only the reaction steps in black). top row Dependency of peak open probability $(\mathrm{Po})$ on the duration of monoexponential agonist pulses of different concen- tration $(0.1,1$, and $5 \mathrm{mM})$. bottom row Dependency of receptor occupancy $(\mathrm{Po})$ on the amplitude of a $1 \mathrm{~ms}$ long agonist transient. The insets display examples of simulated receptor responses to different agonist pulses. All simulations were run in ChanneLab (Synaptosolf, Inc.), using a Runge-Kutta 4 integration

currents, their predictive values is inevitably hampered by uncertainties associated with most of the parameters that are being used. Within a certain cell population, for example, there can be such a wide range of synapses differing in their structure, size, number and distribution of receptors, release properties, and glial coverage [5-10], that the derived "average" neurotransmitter profile may occur only at a small fraction of all synaptic contacts.

It is also worth noting that, to some extent, the neurotransmitter profile concentration varies with the distance from an active release site, as illustrated in Fig. 3. In most instances, it does not perfectly follow the profile of a simple monoexponential function (particularly long after release has occurred). Therefore, the notional measurements of the neurotransmitter concentration profile in the cleft often represent an averaged spatiotemporal concentration profile across the whole postsynaptic region, where the receptors contributing to synaptic responses reside. It should not be interpreted as a faithful representation of the neurotransmitter concentration profile experienced by all the receptors distributed anywhere across the entire postsynaptic area.

\section{The Competitive Low-affinity Antagonist Method}

The use of competitive antagonists with a fast unbinding rate for estimating the time course of neurotransmitter in the cleft was introduced by Clements et al. [105], who estimated the glutamate transient at excitatory synapses in cultured hippocampal neurons using a low-affinity compet-
Although these approaches can be used to explore role of morphological and kinetic variables on synaptic 
a

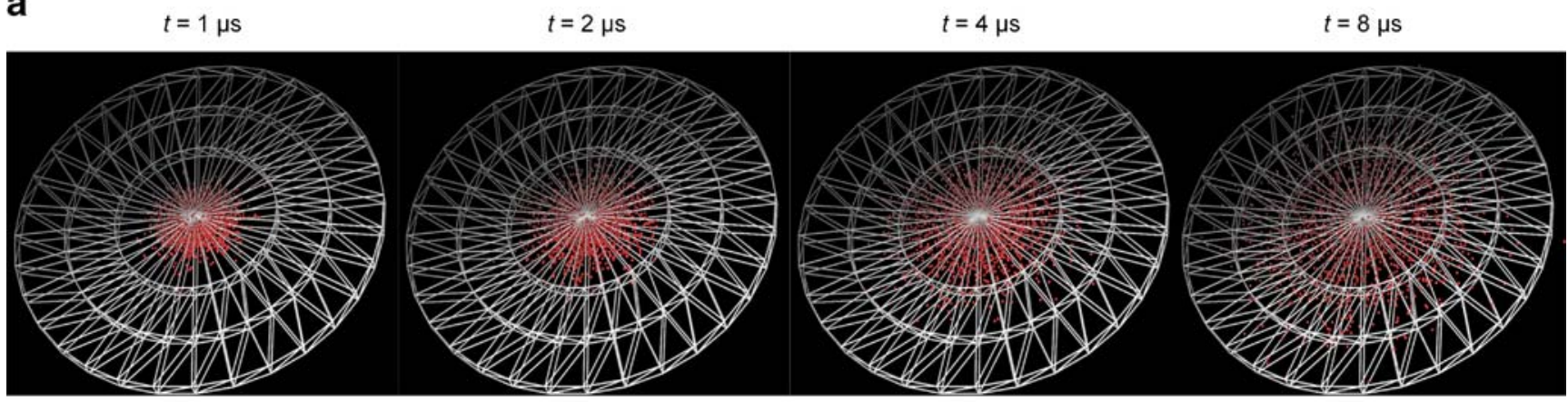

b

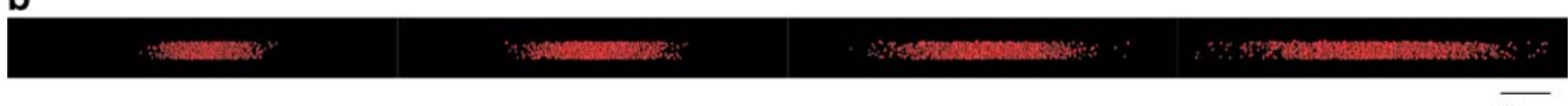

c

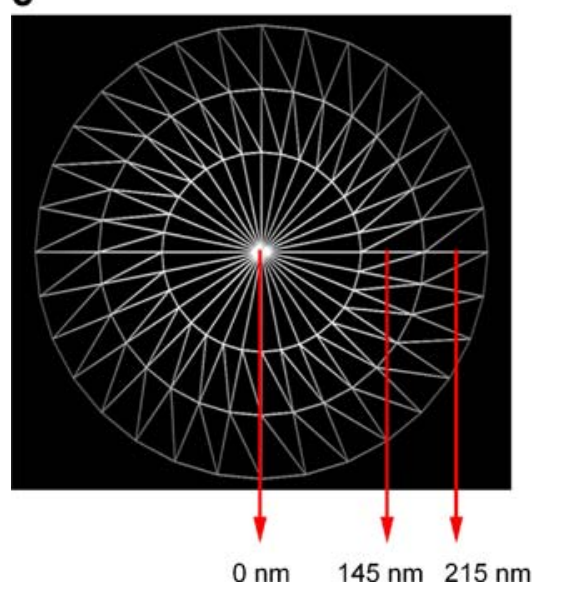

Fig. 3 Monte Carlo simulation of neurotransmitter diffusion in a disk. The neurotransmitter (red dots) diffuses in a disk (height $h=20 \mathrm{~nm}$, radius $r=250 \mathrm{~nm}$, white wireframes) representing the apposition zone between pre and postsynaptic terminals (i.e., the cleft). At $t=0,2000$ neurotransmitter molecules are released from a point source at the center of the disk, and diffuse with an apparent diffusion coefficient of $3.3 \mathrm{~cm}^{2} / \mathrm{s}$ [80]. a Snapshots of neurotransmitter diffusion 1-2-4-8 $\mathrm{s}$ after release. b Side view of neurotransmitter diffusion in the cleft, at the times indicated in a. c Top view of the apposition zone. This is

itive NMDAR antagonist. When postsynaptic receptors are in equilibrium with the antagonist at a $K_{\mathrm{d}}$ concentration, on average half of them are occupied by the drug (assuming there is only one antagonist binding site on each receptor). If the average lifetime of the antagonist-receptor bound complex is much longer than the length of the agonist pulse, the response in the presence of the antagonist is half of that observed in control. If, however, the unbinding rate of the antagonist is comparable with the length of the agonist pulse, at least some antagonist molecules unbind and leave the receptor available for binding the agonist and eventually open. In this case, two predictions can be made: first, the current response is less inhibited than calculated at equilibrium. Second, the rise time of the current response is slowed down in the presence of antagonist, because at least divided in three concentric disks and annuli, with outer radii $r=110$ $180-250 \mathrm{~nm}$, respectively. The red arrows indicate the mean distance between each disk or annulus and the release site. d Examples of the glutamate waveforms monitored in each one of the three volumes described in c. Each trace represents the average of 100 Monte Carlo runs, iterated for $10^{4}$ times with $\Delta t=1 \mu \mathrm{s}$. The geometry was created in silico with an open source program (Blender), imported in a Monte Carlo simulation environment (MCell) and rendered with the visualization software DReAMM [143, 144]

some agonist molecules bind (and activate) the receptor only after the antagonist molecules are unbound. Both predictions are confirmed by direct experimental observations in excised patches using antagonists for NMDARs [105], AMPARs [88], GABA ${ }_{\mathrm{A}}$ Rs [106], and glycine receptors [107]. More importantly, the amount of inhibition depends on the actual profile of transmitter in the cleft: the longer the receptors are exposed to the agonist, the more antagonist molecules unbind and are replaced by agonist. In the case of glutamate and glycine receptors, a full doseinhibition curve of synaptic currents is available [98, 105, 107]. The data are fitted using only the peak agonist concentration and its clearance time as free parameters and the best fits are obtained with agonist pulses of at least $1 \mathrm{mM}$ and shorter than $1 \mathrm{~ms}$. 
This method is rather ingenious and can be implemented with technically straightforward experiments. However, there are a number of uncertainties for its application: first, this approach relies on the exact knowledge of the kinetic properties of postsynaptic receptors and the values for the binding and unbinding rates of the antagonist. The first can be obtained either from the kinetic analysis of single channel recordings [108, 109] or from the analysis of macroscopic currents exposed to a fast agonist transient $[105,110,111]$. While the single-channel approach allows estimate of several rate constants even in models with many different kinetic states [109, 112], it does not provide information on the kinetics of desensitization, for example. In addition, single-channel experiments can only be performed on channels whose conductance is big enough to allow good time resolution, like glycine receptors [109, 113] or NMDARs [114], having limited utility for channels with lower conductance $\left(\mathrm{GABA}_{\mathrm{A}} \mathrm{R}\right.$ and AMPARs), for which ultra-fast concentration jumps are preferred. However, due to the high time correlation between adjacent points [115] only few parameters can be estimated with this method, although a number of different experimental protocols can provide enough information to determine the rates in a simplified model that can give a good description of the experimental results. It is worth noting, however, that the estimates of these kinetic rates are generally obtained from extrasynaptic receptors, since access to synaptic ones is hard to achieve (but see [116]). This raises the possibility of differences between the two populations of receptors (the ones activated during synaptic events and those studied for kinetics analysis) that could be due to different subunit composition, the modulatory effects of anchoring proteins, intracellular kinases and phosphatases that are lost following patch excision or the effect of channel density [117], all factors that are difficult to assess experimentally.

Furthermore, in order to limit the number of fitted parameters, the transmitter pulse is generally modeled as an exponential function, while the actual neurotransmitter time profile obtained from solving diffusion equations may deviate from this simplified profile (see also Fig. 3). The exponential approximation used by most authors, however, allows for a straightforward comparison of the various available estimates. The most notable limitation of the competitive low-affinity antagonist approach is that for a given concentration of antagonist, the same amount of inhibition of the control response can be observed with either a short pulse of high agonist concentration or a long pulse of low agonist concentration [98]. In the first case, few antagonist molecules unbind during the (short) pulse, but they are quickly replaced by the agonist present at high concentration. In the opposite case, there are a lot more antagonist molecules that unbind during the (long) pulse, but they are effectively replaced by agonist molecules over the course of the prolonged low-concentration transient. This limitation (first indicated by Diamond [98]) can be observed in the top row of Fig. 4, where the values of peak agonist concentration and decay time that give a $50 \%$ inhibition at $K_{\mathrm{d}}$ concentration of a fast competitive antagonist are calculated for published kinetic models of NMDARs [105], AMPARs [88], glycine receptors [107], and for a standard del Castillo-Katz model [118] (see Fig. 1 for details on the kinetic rates of each model). It is clear that very different concentration time courses give rise to the same level of observed inhibition for all four different receptors. This feature is entirely independent of the exact details of the model (number of binding sites, open states, intermediate shut states, and desensitization rates all differ in the models included in the figure), and accordingly is observed with the simplest model where agonist and antagonist compete for a single binding site (last column of Fig. 4).

When the results of experiments performed with many antagonist concentrations are put together the fit of the resulting dose-inhibition curve with the peak agonist concentration and decay time as free parameters does not resolve this ambiguity. To prove this, the responses to an agonist pulse of $1 \mathrm{mM}$ with $\tau_{\text {decay }}=1 \mathrm{~ms}$ are calculated from the $Q$ matrices (where $Q$ is defined as the matrix containing the transition rates to and from all states present in the model [119]) corresponding to the four models of Fig. 1 by solving with an adaptive Runge-Kutta integration (RK5) the differential equations that describe the current time course [120]:

$\frac{d \vec{P}(t)}{d t}=\vec{P}(t) \times Q(t)$

where $\vec{P}(t)$ is the row vector of occupancies of each of the states of the model and $Q(t)$ is the matrix with the transition rates. The current amplitude is calculated as the sum of the occupancies of the open states. A dose-inhibition curve is determined for seven antagonist concentrations (attaching a $7 \%$ error to each calculated point, in line with previously published data $[98,105,107])$. The time course of agonist in the cleft $(A(t))$ is described by the function:

$A(t)=\left\{\begin{array}{l}0 \cdots \cdots+\cdots \cdots \cdots \text { if } \cdots t<0 \\ A_{\text {peak }} \times \mathrm{e}^{-\frac{t}{\tau}} \cdots \text { if } \cdots t \geq 0\end{array}\right.$

and the values of $A_{\text {peak }}$ and $\tau_{\text {decay }}$ that give the best fit of the dose-inhibition curve are determined with a fitting routine written in Interactive Data Language (IDL, from ITT Visual Information solutions, Boulder, Colorado) that uses a Levenberg-Marquardt algorithm to minimize the sum of squared deviation (SSD) from the data points. 


\section{NMDAR \\ Clements et al (1992) \\ AMPAR \\ Diamond and Jahr (1997) \\ GlyR \\ Beato (2008) \\ Del Castillo and Katz (1957)}

$50 \%$ inhibition curves of simulated patch currents at $K_{d}$ concentration of low-affinity competitive antagonists
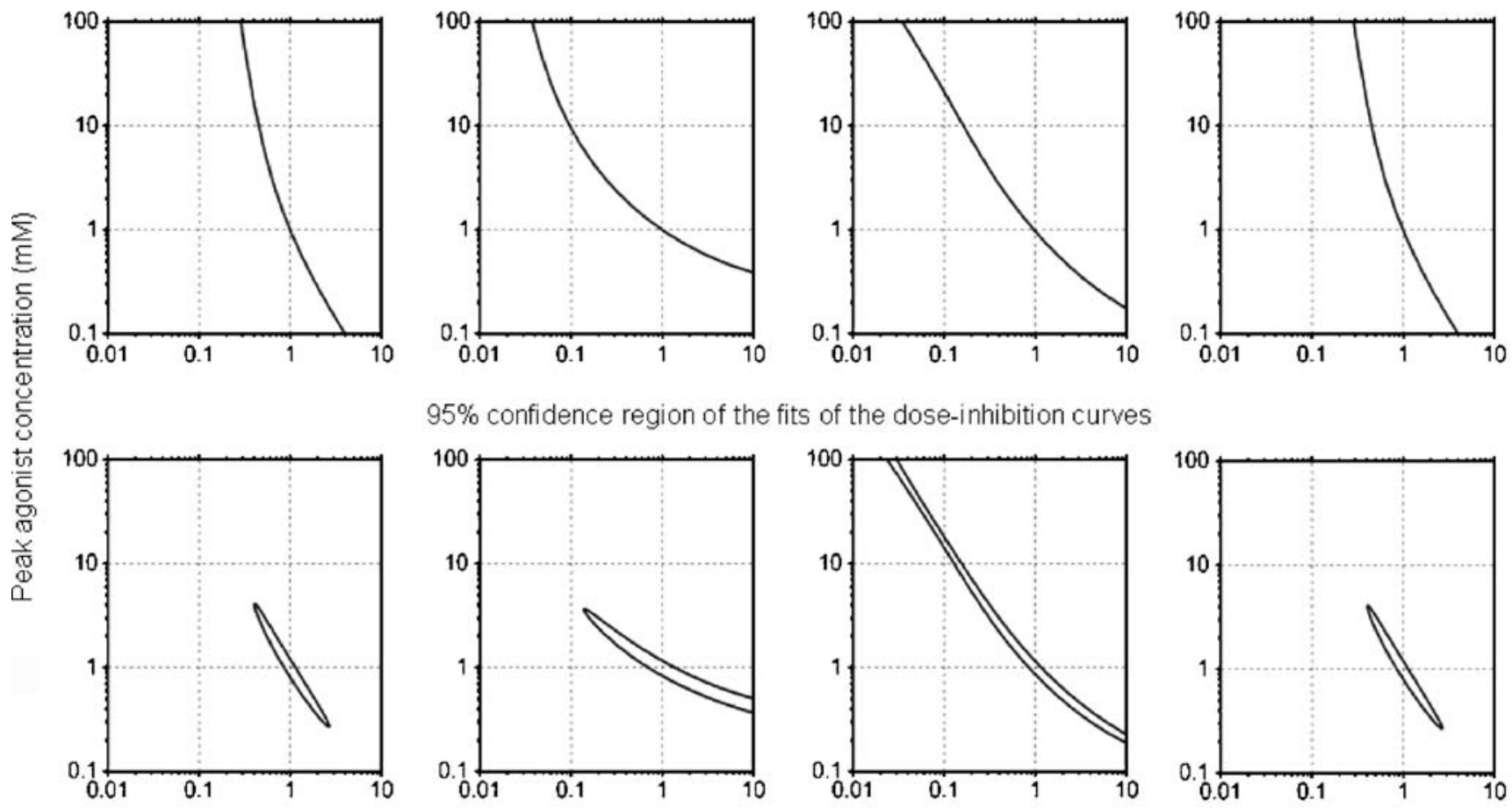

$95 \%$ confidence region of the fits of the dose-inhibition curves
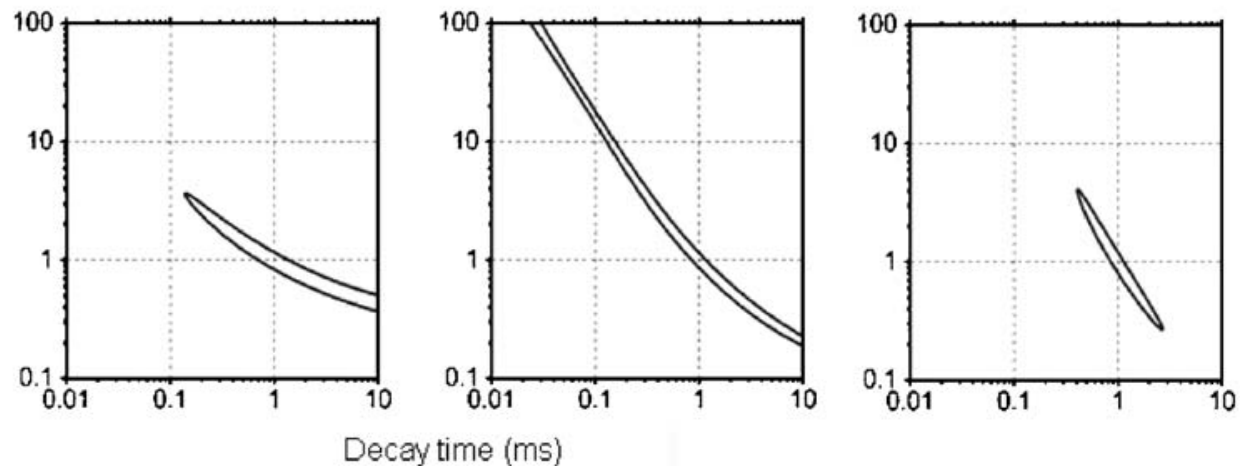

Fig. 4 The peak concentration and time course of neurotransmitter in the cleft is not unambiguously determined by low-affinity antagonists. top row The continuous line in the plots shows the combined values of neurotransmitter peak concentration and decay time that can give rise to $50 \%$ inhibition by low-affinity antagonists. For each receptor kinetic model, we calculated a dose-inhibition curve with seven concentrations of antagonists, for a $1 \mathrm{mM} \cdot 1 \mathrm{~ms}$ agonist pulse. The dose-inhibition curve was then fitted using the peak agonist

The fitting routine invariably converges to the correct values ( $\pm 1 \%$ typically). However, the error on the calculated estimates is always bigger than one order of magnitude (in a logarithmic scale) for both parameters. This is because the two variables are heavily correlated ( $r$ varying between 0.996 and 0.999 among the four models tested). We have therefore determined the region of the two-dimensional parameter space in which the values of the SSD deviates from the minimum by less than $t^{2}$, where $t=2.57$ is the value of the Student's $t$ statistic with 5 degrees of freedom (the seven simulated data points minus the two estimated parameters) that corresponds to the arbitrarily chosen $95 \%$ confidence limit. In other words, all the parameters included in this region provide an equally good fit of the dose-inhibition curve. As seen in the plots in the bottom row of Fig. 4, for all four models, the possible parameters describing the concentration profile extend over most of the parameter space in which the search is performed and are therefore completely undetermined. concentration and decay time as the only free parameters. The best fit was invariably obtained for values very close to $1 \mathrm{mM} 1 \mathrm{~ms}$. bottom row Calculated 95\% confidence limit for each fit. The fits span a 1- or 2-orders-of-magnitude range around the best parameters, with a high correlation coefficient $(>0.99)$. This implies that despite convergence of the fitting routine, the two fitted parameters (i.e., neurotransmitter peak concentration and decay time) are substantially undetermined

The method of the competitive low-affinity antagonist alone cannot produce unambiguous results, even when the number of fitted parameters is limited to two. In order to overcome this problem, Overstreet et al. [106] estimated that GABA reaches a cleft concentration of $3-5 \mathrm{mM}$, that decays with $\tau_{\text {decay }}=0.3-0.6 \mathrm{~ms}$, using a single antagonist concentration and performing a fit of the whole time course of GABAergic IPSCs. With this method, information contained in the amplitude variation and in the change in rise time induced by the antagonist is simultaneously included. As a result, the confidence limits in the estimates of the two parameters are much narrower and no correlation is apparent (see Fig. 16.6B in [106]). One possible limitation of this approach is that it relies on a perfect match between the kinetic properties of synaptic receptors and the extrasynaptic ones that are used for determining the kinetic rates that we mentioned [121]. Furthermore, especially in brain slices (as opposed to cultured neurons), the observed rise times of PSCs are affected by the intrinsic 
filtering of the recording apparatus (parallel circuit of series resistance and whole cell capacitance) as well as by the cable filtering, which is more severe for release sites that are farther away from the soma. Therefore, fitting the time course of observed PSCs to a kinetic scheme has some intrinsic limitations, especially for fast currents like AMPAR or glycine mediated ones. In [88], the authors established the glutamate concentration in the cleft by combining information from the dose-inhibition curve and the changes in rise time observed with the antagonist and/or with a blocker of glutamate transport. Through this approach, it was shown that the glutamate transient is best described by a double exponential waveform with peak concentrations of 2.7 and $0.4 \mathrm{mM}$ and with $\tau_{\text {decay }}=0.1 \mathrm{~ms}$ and $\tau_{\text {decay }}=2.1 \mathrm{~ms}$, respectively. Blocking transporters has the advantage that it can change the clearance time with negligible effects on the neurotransmitter peak concentration and therefore can be assumed to alter independently one of the two correlated parameters. Accordingly, the comparison of inhibition before and after block of transporters has been instrumental in determining the time course for glutamate at AMPARs [88] and of glycine at synapses onto motoneurons in the spinal cord [107].

A different but conceptually similar method has been used for determining the time course of synaptically released GABA in cultured hippocampal neurons. Instead of partially occupying the agonist binding sites with an antagonist, the authors used modulators like chlorpromazine or zinc $[122,123]$ or $\mathrm{pH}$ manipulations [110] that affect (mostly) the agonist binding rates. These manipulations, as noticed by the authors, affect macroscopic currents evoked by ultra-fast application of transmitter differently from synaptic currents. This is attributed to differences between the time course (and in particular $\tau_{\text {decay }}$ ) of the ultra-fast application and that of synaptically released GABA. This discrepancy, together with the determination of the kinetic rates before and after application of the modulatory agent, allows determining the time course of GABA release. The estimate still relies on an exact description of the kinetics of postsynaptic receptors. However, its goodness is strongly supported by the fact that very similar values for the peak concentration and $\tau_{\text {decay }}(2-$ $3 \mathrm{mM}$ and $0.1 \mathrm{~ms}$, respectively) are estimated with the three independent approaches (i.e., chlorpromazine, zinc, and variations in $\mathrm{pH}$ ).

\section{Sniffers and Imaging Approaches}

Various attempts have been made to develop glutamate sensors as reporters of neurotransmitter release. Excised patches of cell membranes enriched with high-affinity receptors, for example, have been used to probe the identity of the neurotransmitter released from retinal bipolar cells [124, 125]. The power of this clever design, which in principle can offer a highly sensitive and temporally precise read-out of the glutamate transient (depending on the properties of the receptors in the "sniffer-patch"), is once again hampered by the relative inaccessibility of the cleft region at central synapses.

Although the development of such an ideal experimental tool is still in progress, a number of promising attempts have been made taking advantage of biochemical engineering and high-resolution optical detection methods. The trick here is to have fast and sensitive glutamate reporters combined with high temporal and spatial resolution detection of the glutamate transient, but as it might be expected, the real scenario involves some trade-offs between all these parameters.

Namiki et al. [126] developed an optical glutamate probe (glutamate (E) optical sensor, EOS) based on a mutated AMPAR GluR2 S1S2 ligand-binding domain fused with the Oregon green dye. The conformational change of the ligand-binding domain induced by glutamate leads to $40 \%$ increase in the maximal fluorescence intensity of Oregon green, at the peak of its emission wavelength $(520 \mathrm{~nm})$. The kinetics of the reaction is dictated by the rate of glutamate binding to the S1S2 domain $\left(10^{7} \mathrm{M}^{-1} \mathrm{~s}^{-1}\right.$, if this remains comparable to that of native S1S2 domains) and the measured off-rate $\left(0.67 \mathrm{~s}^{-1}\right)$ [126]. The slow dissociation kinetics, together with a relatively low $\mathrm{EC}_{50}(148 \mathrm{nM})$, limit the application of EOS to the late and prolonged phases of neurotransmitter release. In addition, anchoring of EOS to the cell membrane through a generic biotinstreptavidin reaction currently precludes any cell-specific expression of this reporter.

Some of these limitations can be potentially overcome by using optical probes that are genetically encoded, and that at least in principle can be fused with proteins selectively expressed in synaptic or non-synaptic regions. Recent examples of these genetically encoded optical probes are the glutamate-sensing fluorescent reporter (GluSnFR) [127], the more sensitive variant SuperGluSnFR [128], or the fluorescence indicator protein for glutamate (FLIPE) [129]. The general design of these reporters involves fusion of a cyan and yellow fluorescent protein (CFP and YFP, respectively) to the periplasmic glutamatebinding protein from E. coli (GltI), and a truncated PDGF receptor that ensures membrane anchoring of the construct [128]. The CFP and YFP are chosen because they currently represent the best pair of fluorescent proteins enabling fluorescence resonance energy transfer (FRET) [127]. The conformational change in the S1S2 domain induced by glutamate binding brings the two fluorophores closer to each other $(<80 \AA)$ leading to the non-radiative energy transfer from CFP to YFP detected in FRET experiments 
(the emission spectrum of CFP significantly overlaps with the excitation spectrum of YFP). A series of mutations of this construct has significantly improved its affinity for glutamate and the extent of CFP/YFP emission ratio upon glutamate binding (in Ringer solution, $K_{\mathrm{d}}=2.5 \mu \mathrm{M}$ and $44 \%$ change in CFP/YFP emission [128]). The estimated kinetic properties of SuperGluSnFR, however, seem best suited for detecting small and slow glutamate transients like those occurring during the tail of a synaptic event or during spillover-activation of nearby receptors, as opposed to those that are attained in the synaptic cleft region. For example, in cultured hippocampal neurons, the glutamate transient reported by SuperGluSnFR has a $20-80 \%$ rise time $=$ $6.6 \mathrm{~ms},[\mathrm{Glu}]_{\text {peak }}=720 \mathrm{nM}, \tau_{\text {decay }}=40 \mathrm{~ms}$, but the subcellular resolution of the signal is limited by the camera resolution.

A wide range of imaging techniques could also provide useful quantitative insights into the diffusion properties of various molecules inside and outside the synaptic cleft [130]. For example, fluorescence recovery after photobleaching (FRAP), fluorescence loss in photobleaching (FLIP), and fluorescence localization after photoactivation (FLAP) could, in principle, be used to estimate the characteristics of neurotransmitter diffusion among distinct subcellular compartments.

With FRAP, the experimental preparation is first equilibrated with a fluorophore, which is then bleached in a small region with a high-intensity light source (e.g., a laser). The rate and extent of fluorescence recovery can be followed in time, providing information on the fluorophore diffusion between the bleached region and neighboring ones. This method has been used to analyze inhomogeneities in the diffusion coefficient of small and large particles within the cytoplasm [131]. A modified but related approach consists of constantly bleaching the fluorophore at a particular site and monitoring the FLIP at a nearby location to examine the exchange in fluorescence between the bleached and neighboring sites. If the fluorophore has similar biophysical properties to those of the neurotransmitter of interest, one could possibly determine how far and how quickly it travels away from the bleached area [132]. The general principles of these two techniques are also shared by FLAP, where one follows the localization of a photoactivated molecule, like PA-GFP [133] or Dronpa [134], or of a fluorescent molecule that changes its spectrum of emission upon exposure to UV light (e.g., Kaede [135]). The advantage of these techniques is that they can be used in confocal or two-photon microscopy setups, but are limited, however, by the temporal and spatial resolution of these systems.

That is why the development of other techniques has become so crucial. Particularly noteworthy are fluorescence lifetime imaging (FLIM) and fluorescence correlation spectroscopy (FCS) [136], which have sub-microsecond time limits, or photoactivated localization microscopy (PALM) and single-particle tracking PALM (sptPALM) [137], which significantly enhance the spatial resolution of the signal emitted by labeled molecules [138, 139]. With FLIM, one measures the exponential decay in the emission intensity of a fluorophore (i.e., its lifetime). This measure is independent of the fluorophore concentration, but changes if the environment surrounding the fluorophore (e.g., polarity, ion concentration, and viscosity) alters its electronic state. Fluorescence lifetimes can be measured in the time and frequency domains, and have been used in biological systems to measure ionic concentrations $\left(\mathrm{Ca}^{2+}\right.$, $\mathrm{Cl}^{-}, \mathrm{O}_{2}$ ) or the subcellular localization of various macromolecules [140]. This technique can also be combined with FCS [141] to determine the diffusion coefficient of fluorescent molecules within a known volume [140]. An interesting variation of FCS, scanning FCS (sFCS), does not require a priori knowledge of the measurement volume, and therefore is probably better suited for diffusion estimates in living systems [142].

A recently developed approach, sptPALM, has enabled tracking of single fluorescent protein chimeras with nanometer spatial resolution. This allows one to obtain maps of single-molecule diffusion that can be useful to study cell dynamics, as well as the properties of subcellular microenvironments. It would be of interest to test whether this kind of approach could be used to study the diffusion profile of smaller molecules, like neurotransmitters, particularly in living tissues. These and other techniques are constantly being refined and their application to synaptic physiology is expected to enable the investigation of aspects of synaptic transmission and neurotransmitter diffusion that are currently precluded by indirect pharmacological and modeling approaches.

\section{Conclusions}

The problem of the determination of the time course of transmitter in the cleft has spanned more than 60 years of neuroscience research and a number of direct and indirect approaches have been used to determine this elusive variable. Despite all the efforts, we are still far from a definite consensus, even for the most studied synapses in the central nervous system. Old techniques and innovative approaches have been devised that are continuously challenged by the advent of improved technologies that can resolve events on a faster time scale and with better spatial resolution. In this review, we highlighted the advantages and pitfalls of each method that has been used in the past and analyzed the potential of new optical techniques that in the future could provide quantitative 
descriptions of the fundamental mechanisms underlying the dynamics of inter-neuronal signaling. It is likely that refinement of these novel approaches will ultimately answer many open questions that have haunted neuroscientists since the demonstration of quantal release of neurotransmitter as the main form of communication between neuronal cells.

Acknowledgments A.S. is supported by the NINDS Intramural Research Program and Human Frontier Science Program (RGP50/ 2006). M.B. is a Royal Society University Research Fellow and recipient of a Wellcome Trust Grant (Project Grant 07662/Z/05/A). The Authors would like to thank D.M. Kullmann, K.A. Pelkey and L. $\mathrm{G}$. Wu for valuable comments on the manuscript.

Open Access This article is distributed under the terms of the Creative Commons Attribution Noncommercial License which permits any noncommercial use, distribution, and reproduction in any medium, provided the original author(s) and source are credited.

\section{References}

1. Banks MI, Li TB, Pearce RA (1998) The synaptic basis of GABAA, slow. J Neurosci 18(4):1305-1317

2. Lu T, Rubio ME, Trussell LO (2008) Glycinergic transmission shaped by the corelease of GABA in a mammalian auditory synapse. Neuron 57(4):524-535

3. Szabadics J, Tamas G, Soltesz I (2007) Different transmitter transients underlie presynaptic cell type specificity of GABAA, slow and GABAA, fast. Proc Natl Acad Sci U S A 104 (37):14831-14836

4. Szapiro G, Barbour B (2007) Multiple climbing fibers signal to molecular layer interneurons exclusively via glutamate spillover. Nat Neurosci 10(6):735-742

5. Huang EP, Stevens CF (1997) Estimating the distribution of synaptic reliabilities. J Neurophysiol 78(6):2870-2880

6. Nusser Z, Lujan R, Laube G, Roberts JDB, Molnar E, Somogyi P (1998) Cell type and pathway dependence of synaptic AMPA receptor number and variability in the hippocampus. Neuron 21(3):545-559

7. Racca C, Stephenson FA, Streit P, Roberts JD, Somogyi P (2000) NMDA receptor content of synapses in stratum radiatum of the hippocampal CA1 area. J Neurosci 20(7):2512-2522

8. Schikorski T, Stevens CF (1997) Quantitative ultrastructural analysis of hippocampal excitatory synapses. J Neurosci 17 (15):5858-5867

9. Takumi Y, Ramirez-Leon V, Laake P, Rinvik E, Ottersen OP (1999) Different modes of expression of AMPA and NMDA receptors in hippocampal synapses. Nat Neurosci 2(7):618-624

10. Ventura R, Harris KM (1999) Three-dimensional relationships between hippocampal synapses and astrocytes. J Neurosci 19 (16):6897-6906

11. Acheson GH (1948) Physiology of neuro-muscular junctions; chemical aspects. Fed Proc 7(3):447-457

12. Krnjevic K, Miledi R (1958) Acetylcholine in mammalian neuromuscular transmission. Nature 182(4638):805-806

13. Kuffler SW, Yoshikami D (1975) The number of transmitter molecules in a quantum: an estimate from iontophoretic application of acetylcholine at the neuromuscular synapse. J Physiol 251(2):465-482

14. Hartzell HC, Kuffler SW, Yoshikami D (1975) Post-synaptic potentiation: interaction between quanta of acetylcholine at the skeletal neuromuscular synapse. J Physiol 251(2):427-463
15. Matthews-Bellinger J, Salpeter MM (1978) Distribution of acetylcholine receptors at frog neuromuscular junctions with a discussion of some physiological implications. J Physiol 279:197-213

16. Wightman RM, Jankowski JA, Kennedy RT, Kawagoe KT, Schroeder TJ, Leszczyszyn DJ, Near JA, Diliberto EJ Jr, Viveros OH (1991) Temporally resolved catecholamine spikes correspond to single vesicle release from individual chromaffin cells. Proc Natl Acad Sci U S A 88(23):10754-10758

17. Pothos EN, Davila V, Sulzer D (1998) Presynaptic recording of quanta from midbrain dopamine neurons and modulation of the quantal size. J Neurosci 18(11):4106-4118

18. Burger PM, Mehl E, Cameron PL, Maycox PR, Baumert M, Lottspeich F, De Camilli P, Jahn R (1989) Synaptic vesicles immunoisolated from rat cerebral cortex contain high levels of glutamate. Neuron 3(6):715-720

19. Riveros N, Fiedler J, Lagos N, Munoz C, Orrego F (1986) Glutamate in rat brain cortex synaptic vesicles: influence of the vesicle isolation procedure. Brain Res 386(1-2):405-408

20. Takamori S, Holt M, Stenius K, Lemke EA, Grønborg M, Riedel D, Urlaub H, Schenck S, Brügger B, Ringler P, Müller SA, Rammner B, Gräter F, Hub JS, De Groot BL, Mieskes G, Moriyama Y, Klingauf J, Grubmüller H, Heuser J, Wieland F, Jahn R (2006) Molecular anatomy of a trafficking organelle. Cell 127(4):831-846

21. Harata N, Ryan TA, Smith SJ, Buchanan J, Tsien RW (2001) Visualizing recycling synaptic vesicles in hippocampal neurons by FM 1-43 photoconversion. Proc Natl Acad Sci U S A 98 (22):12748-12753

22. Bekkers JM, Richerson GB, Stevens CF (1990) Origin of variability in quantal size in cultured hippocampal neurons and hippocampal slices. Proc Natl Acad Sci U S A 87(14):5359-5362

23. Sulzer D, Edwards R (2000) Vesicles: equal in neurotransmitter concentration but not in volume. Neuron 28(1):5-7

24. Wu XS, Xue L, Mohan R, Paradiso K, Gillis KD, Wu LG (2007) The origin of quantal size variation: vesicular glutamate concentration plays a significant role. J Neurosci 27(11):3046-3056

25. Daniels RW, Collins CA, Chen K, Gelfand MV, Featherstone DE, DiAntonio A (2006) A single vesicular glutamate transporter is sufficient to fill a synaptic vesicle. Neuron 49(1):11-16

26. Schikorski T, Stevens CF (2001) Morphological correlates of functionally defined synaptic vesicle populations. Nat Neurosci 4 (4):391-395

27. Sorra KE, Harris KM (1993) Occurrence and three-dimensional structure of multiple synapses between individual radiatum axons and their target pyramidal cells in hippocampal area CA1. J Neurosci 13(9):3736-3748

28. Gulyas AI, Miles R, Sík A, Tóth K, Tamamaki N, Freund TF (1993) Hippocampal pyramidal cells excite inhibitory neurons through a single release site. Nature 366(6456):683-687

29. Triller A, Korn H (1982) Transmission at a central inhibitory synapse. III. Ultrastructure of physiologically identified and stained terminals. J Neurophysiol 48(3):708-736

30. Auger C, Kondo S, Marty A (1998) Multivesicular release at single functional synaptic sites in cerebellar stellate and basket cells. J Neurosci 18(12):4532-4547

31. Korn H, Sur C, Charpier S, Legendre P, Faber DS (1994) The one-vesicle hypothesis and multivesicular release. Adv Second Messenger Phosphoprotein Res 29:301-322

32. Christie JM, Jahr CE (2006) Multivesicular release at Schaffer collateral-CA1 hippocampal synapses. J Neurosci 26(1):210-216

33. Conti R, Lisman J (2003) The high variance of AMPA receptorand NMDA receptor-mediated responses at single hippocampal synapses: evidence for multiquantal release. Proc Natl Acad Sci U S A 100(8):4885-4890

34. Foster KA, Crowley JJ, Regehr WG (2005) The influence of multivesicular release and postsynaptic receptor saturation on 
transmission at granule cell to Purkinje cell synapses. J Neurosci 25(50):11655-11665

35. Korn H, Bausela F, Charpier S, Faber DS (1993) Synaptic noise and multiquantal release at dendritic synapses. J Neurophysiol 70 (3): 1249-1254

36. Oertner TG, Sabatini BL, Nimchinsky EA, Svoboda K (2002) Facilitation at single synapses probed with optical quantal analysis. Nat Neurosci 5(7):657-664

37. Prange O, Murphy TH (1999) Analysis of multiquantal transmitter release from single cultured cortical neuron terminals. J Neurophysiol 81(4):1810-1817

38. Singer JH, Lassova L, Vardi N, Diamond JS (2004) Coordinated multivesicular release at a mammalian ribbon synapse. Nat Neurosci 7(8):826-833

39. Tong G, Jahr CE (1994) Multivesicular release from excitatory synapses of cultured hippocampal neurons. Neuron 12(1):51-59

40. Wadiche JI, Jahr CE (2001) Multivesicular release at climbing fiber-Purkinje cell synapses. Neuron 32(2):301-313

41. Higley MJ, Soler-Llavina GJ, Sabatini BL (2009) Cholinergic modulation of multivesicular release regulates striatal synaptic potency and integration. Nat Neurosci 12(9):1121-1128

42. Liu G, Choi S, Tsien RW (1999) Variability of neurotransmitter concentration and nonsaturation of postsynaptic AMPA receptors at synapses in hippocampal cultures and slices. Neuron 22 (2):395-409

43. Asztely F, Erdemli G, Kullmann DM (1997) Extrasynaptic glutamate spillover in the hippocampus: dependence on temperature and the role of active glutamate uptake. Neuron 18(2):281-293

44. Ceccarelli B, Hurlbut WP, Mauro A (1972) Depletion of vesicles from frog neuromuscular junctions by prolonged tetanic stimulation. J Cell Biol 54(1):30-38

45. Heuser JE, Reese TS (1973) Evidence for recycling of synaptic vesicle membrane during transmitter release at the frog neuromuscular junction. J Cell Biol 57(2):315-344

46. Chow RH, von Ruden L, Neher E (1992) Delay in vesicle fusion revealed by electrochemical monitoring of single secretory events in adrenal chromaffin cells. Nature 356(6364):60-63

47. He L, Wu XS, Mohan R, Wu LG (2006) Two modes of fusion pore opening revealed by cell-attached recordings at a synapse. Nature 444(7115):102-105

48. Harata NC, Choi S, Pyle JL, Aravanis AM, Tsien RW (2006) Frequency-dependent kinetics and prevalence of kiss-and-run and reuse at hippocampal synapses studied with novel quenching methods. Neuron 49(2):243-256

49. Zhang Q, Li Y, Tsien RW (2009) The dynamic control of kissand-run and vesicular reuse probed with single nanoparticles. Science 323(5920):1448-1453

50. Fernandez JM, Neher E, Gomperts BD (1984) Capacitance measurements reveal stepwise fusion events in degranulating mast cells. Nature 312(5993):453-455

51. Neher E, Marty A (1982) Discrete changes of cell membrane capacitance observed under conditions of enhanced secretion in bovine adrenal chromaffin cells. Proc Natl Acad Sci U S A 79 (21):6712-6716

52. Almers W, Breckenridge LJ, Iwata A, Lee AK, Spruce AE, Tse FW (1991) Millisecond studies of single membrane fusion events. Ann N Y Acad Sci 635:318-327

53. Spruce AE, Breckenridge LJ, Lee AK, Almers W (1990) Properties of the fusion pore that forms during exocytosis of a mast cell secretory vesicle. Neuron 4(5):643-654

54. Aravanis AM, Pyle JL, Tsien RW (2003) Single synaptic vesicles fusing transiently and successively without loss of identity. Nature 423(6940):643-647

55. Gandhi SP, Stevens CF (2003) Three modes of synaptic vesicular recycling revealed by single-vesicle imaging. Nature 423 (6940):607-613
56. Balaji J, Ryan TA (2007) Single-vesicle imaging reveals that synaptic vesicle exocytosis and endocytosis are coupled by a single stochastic mode. Proc Natl Acad Sci U S A 104 (51):20576-20581

57. Granseth B, Odermatt B, Royle SJ, Lagnado L (2006) Clathrinmediated endocytosis is the dominant mechanism of vesicle retrieval at hippocampal synapses. Neuron 51(6):773-786

58. Chen X, Barg S, Almers W (2008) Release of the styryl dyes from single synaptic vesicles in hippocampal neurons. J Neurosci 28(8):1894-1903

59. Renden R, von Gersdorff H (2007) Synaptic vesicle endocytosis at a CNS nerve terminal: faster kinetics at physiological temperatures and increased endocytotic capacity during maturation. J Neurophysiol 98(6):3349-3359

60. Zhang Q, Cao YQ, Tsien RW (2007) Quantum dots provide an optical signal specific to full collapse fusion of synaptic vesicles. Proc Natl Acad Sci U S A 104(45):17843-17848

61. Eccles JC, Jaeger JC (1958) The relationship between the mode of operation and the dimensions of the junctional regions at synapses and motor end-organs. Proc R Soc Lond B Biol Sci 148(930):38-56

62. Katz B, Miledi R (1973) The binding of acetylcholine to receptors and its removal from the synaptic cleft. J Physiol 231 (3):549-574

63. Magleby KL, Stevens CF (1972) The effect of voltage on the time course of end-plate currents. J Physiol 223(1):151-171

64. Eccles JC, Mac FW (1949) Actions of anti-cholinesterases on endplate potential of frog muscle. J Neurophysiol 12(1):59-80

65. Hill AV (1936) Excitation and accommodation in nerve. Proceedings of the Royal Society of London Series B, Biological Sciences 119(814):305-355

66. Eccles JC, Katz B, Kuffler SW (1941) Nature of the "endplate potential" in curarized muscle. J Neurophysiol 4:362-387

67. Nicholson C, Phillips JM (1981) Ion diffusion modified by tortuosity and volume fraction in the extracellular microenvironment of the rat cerebellum. J Physiol 321:225-257

68. Sykova E, Nicholson C (2008) Diffusion in brain extracellular space. Physiol Rev 88(4):1277-1340

69. Nicholson C, Sykova E (1998) Extracellular space structure revealed by diffusion analysis. Trends Neurosci 21(5):207-215

70. Rusakov DA, Kullmann DM (1998) Extrasynaptic glutamate diffusion in the hippocampus: ultrastructural constraints, uptake, and receptor activation. J Neurosci 18(9):3158-3170

71. Tao A, Tao L, Nicholson C (2005) Cell cavities increase tortuosity in brain extracellular space. J Theor Biol 234(4):525-536

72. Hrabe J, Hrabetova S, Segeth K (2004) A model of effective diffusion and tortuosity in the extracellular space of the brain. Biophys J 87(3):1606-1617

73. Hrabetova S, Nicholson C (2004) Contribution of dead-space microdomains to tortuosity of brain extracellular space. Neurochem Int 45(4):467-477

74. Tao L, Nicholson C (2004) Maximum geometrical hindrance to diffusion in brain extracellular space surrounding uniformly spaced convex cells. J Theor Biol 229(1):59-68

75. Nicholson C, Tao L (1993) Hindered diffusion of high molecular weight compounds in brain extracellular microenvironment measured with integrative optical imaging. Biophys J 65 (6):2277-2290

76. Savtchenko LP, Rusakov DA (2005) Extracellular diffusivity determines contribution of high-versus low-affinity receptors to neural signaling. Neuroimage 25(1):101-111

77. Thorne RG, Nicholson C (2006) In vivo diffusion analysis with quantum dots and dextrans predicts the width of brain extracellular space. Proc Natl Acad Sci U S A 103(14):5567-5572

78. Zheng K, Scimemi A, Rusakov DA (2008) Receptor actions of synaptically released glutamate: the role of transporters on the scale from nanometers to microns. Biophys J 95(10):4584-4596 
79. Levin VA, Fenstermacher JD, Patlak CS (1970) Sucrose and inulin space measurements of cerebral cortex in four mammalian species. Am J Physiol 219(5):1528-1533

80. Nielsen TA, DiGregorio DA, Silver RA (2004) Modulation of glutamate mobility reveals the mechanism underlying slowrising AMPAR EPSCs and the diffusion coefficient in the synaptic cleft. Neuron 42(5):757-771

81. Barbour B (2001) An evaluation of synapse independence. J Neurosci 21(20):7969-7984

82. Franks KM, Bartol TM Jr, Sejnowski TJ (2002) A Monte Carlo model reveals independent signaling at central glutamatergic synapses. Biophys J 83(5):2333-2348

83. Kullmann DM, Min MY, Asztely F, Rusakov DA (1999) Extracellular glutamate diffusion determines the occupancy of glutamate receptors at CA1 synapses in the hippocampus. Philos Trans R Soc Lond B Biol Sci 354(1381):395-402

84. Longsworth LG (1953) Diffusion measurements, at 25-degrees, of aqueous solutions of amino acids, peptides and sugars. J Am Chem Soc 75(22):5705-5709

85. Min MY, Rusakov DA, Kullmann DM (1998) Activation of AMPA, kainate, and metabotropic receptors at hippocampal mossy fiber synapses: role of glutamate diffusion. Neuron 21 (3):561-570

86. Pouille F, Scanziani M (2001) Enforcement of temporal fidelity in pyramidal cells by somatic feed-forward inhibition. Science 293(5532):1159-1163

87. Bergles DE, Jahr CE (1997) Synaptic activation of glutamate transporters in hippocampal astrocytes. Neuron 19(6):1297-1308

88. Diamond JS, Jahr CE (1997) Transporters buffer synaptically released glutamate on a submillisecond time scale. J Neurosci 17 (12):4672-4687

89. Wadiche JI, Arriza JL, Amara SG, Kavanaugh MP (1995) Kinetics of a human glutamate transporter. Neuron 14(5):1019-1027

90. Lehre KP, Danbolt NC (1998) The number of glutamate transporter subtype molecules at glutamatergic synapses: chemical and stereological quantification in young adult rat brain. $\mathrm{J}$ Neurosci 18(21):8751-8757

91. Levy LM, Lehre KP, Walaas SI, Storm-Mathisen J, Danbolt NC (1995) Down-regulation of glial glutamate transporters after glutamatergic denervation in the rat brain. Eur J NeuroSci 7 (10):2036-2041

92. Diamond JS (2005) Deriving the glutamate clearance time course from transporter currents in CA1 hippocampal astrocytes: transmitter uptake gets faster during development. J Neurosci 25(11):2906-2916

93. Arnth-Jensen N, Jabaudon D, Scanziani M (2002) Cooperation between independent hippocampal synapses is controlled by glutamate uptake. Nat Neurosci 5(4):325-331

94. Huang YH, Sinha SR, Tanaka K, Rothstein JD, Bergles DE (2004) Astrocyte glutamate transporters regulate metabotropic glutamate receptor-mediated excitation of hippocampal interneurons. J Neurosci 24(19):4551-4559

95. Scimemi A, Fine A, Kullmann DM, Rusakov DA (2004) NR2Bcontaining receptors mediate cross talk among hippocampal synapses. J Neurosci 24(20):4767-4777

96. Brasnjo G, Otis TS (2001) Neuronal glutamate transporters control activation of postsynaptic metabotropic glutamate receptors and influence cerebellar long-term depression. Neuron 31(4):607-616

97. Dehnes Y, Chaudhry FA, Ullensvang K, Lehre KP, Storm-Mathisen J, Danbolt NC (1998) The glutamate transporter EAAT4 in rat cerebellar Purkinje cells: a glutamate-gated chloride channel concentrated near the synapse in parts of the dendritic membrane facing astroglia. J Neurosci 18(10):3606-3619

98. Diamond JS (2001) Neuronal glutamate transporters limit activation of NMDA receptors by neurotransmitter spillover on CA1 pyramidal cells. J Neurosci 21(21):8328-8338
99. Fatt P (1954) Biophysics of junctional transmission. Physiol Rev 34(4):674-710

100. Ogston AG (1955) Removal of acetylcholine from a limited volume by diffusion. J Physiol 128(1):222-223

101. Fatt P, Katz B (1951) An analysis of the end-plate potential recorded with an intracellular electrode. J Physiol 115(3):320-370

102. Bartol TM Jr, Land BR, Salpeter EE, Salpeter MM (1991) Monte Carlo simulation of miniature endplate current generation in the vertebrate neuromuscular junction. Biophys J 59(6):1290-1307

103. Kruk PJ, Korn H, Faber DS (1997) The effects of geometrical parameters on synaptic transmission: a Monte Carlo simulation study. Biophys J 73(6):2874-2890

104. Wahl LM, Pouzat C, Stratford KJ (1996) Monte Carlo simulation of fast excitatory synaptic transmission at a hippocampal synapse. J Neurophysiol 75(2):597-608

105. Clements JD, Lester RA, Tong G, Jahr CE, Westbrook GL (1992) The time course of glutamate in the synaptic cleft. Science 258(5087):1498-1501

106. Overstreet LS, Westbrook GL, Jones MV (2003) Measuring and modeling the spatiotemporal profile of GABA at the synapse. In: Quick MW (ed) Transmembrane transporters. Wiley-Liss, New York, pp 259-276

107. Beato $M$ (2008) The time course of transmitter at glycinergic synapses onto motoneurons. J Neurosci 28(29):7412-7425

108. Beato M, Sivilotti LG (2007) Single-channel properties of glycine receptors of juvenile rat spinal motoneurones in vitro. $\mathrm{J}$ Physiol 580(Pt. 2):497-506

109. Burzomato V, Beato M, Groot-Kormelink PJ, Colquhoun D, Sivilotti LG (2004) Single-channel behavior of heteromeric alphalbeta glycine receptors: an attempt to detect a conformational change before the channel opens. J Neurosci 24 (48):10924-10940

110. Mozrzymas JW, Barberis A, Mercik K, Zarnowska ED (2003) Binding sites, singly bound states, and conformation coupling shape GABA-evoked currents. J Neurophysiol 89(2):871-883

111. Raman IM, Trussell LO (1995) Concentration-jump analysis of voltage-dependent conductances activated by glutamate and kainate in neurons of the avian cochlear nucleus. Biophys J 69(5):18681879

112. Pitt SJ, Sivilotti LG, Beato M (2008) High intracellular chloride slows the decay of glycinergic currents. J Neurosci 28 (45):11454-11467

113. Beato M, Groot-Kormelink PJ, Colquhoun D, Sivilotti LG (2004) The activation mechanism of alphal homomeric glycine receptors. J Neurosci 24(4):895-906

114. Banke TG, Traynelis SF (2003) Activation of NR1/NR2B NMDA receptors. Nat Neurosci 6(2):144-152

115. Celentano JJ, Hawkes AG (2004) Use of the covariance matrix in directly fitting kinetic parameters: application to GABAA receptors. Biophys J 87(1):276-294

116. Lu W, Shi Y, Jackson AC, Bjorgan K, During MJ, Sprengel R, Seeburg PH, Nicoll RA (2009) Subunit composition of synaptic AMPA receptors revealed by a single-cell genetic approach. Neuron 62(2):254-268

117. Legendre P, Muller E, Badiu CI, Meier J, Vannier C, Triller A (2002) Desensitization of homomeric alphal glycine receptor increases with receptor density. Mol Pharmacol 62(4):817-827

118. Del Castillo J, Katz B (1957) Interaction at end-plate receptors between different choline derivatives. Proc R Soc Lond B Biol Sci 146(924):369-381

119. Colquhoun D, Hawkes AG (1982) On the stochastic properties of bursts of single ion channel openings and of clusters of bursts. Philos Trans R Soc Lond B Biol Sci 300(1098):1-59

120. Colquhoun D, Hawkes AG, Merlushkin A, Edmonds B (1997) Properties of single ion channel currents elicited by a pulse of agonist concentration or voltage. Philosophical Transactions of 
the Royal Society of London Series A-Mathematical Physical and Engineering Sciences 355(1730):1743-1786

121. Jones MV, Westbrook GL (1995) Desensitized states prolong GABAA channel responses to brief agonist pulses. Neuron 15 (1):181-191

122. Barberis A, Cherubini E, Mozrzymas JW (2000) Zinc inhibits miniature GABAergic currents by allosteric modulation of GABAA receptor gating. J Neurosci 20(23):8618-8627

123. Mozrzymas JW, Barberis A, Michalak K, Cherubini E (1999) Chlorpromazine inhibits miniature GABAergic currents by reducing the binding and by increasing the unbinding rate of GABAA receptors. J Neurosci 19(7):2474-2488

124. Allen TG (1997) The 'sniffer-patch' technique for detection of neurotransmitter release. Trends Neurosci 20(5):192-197

125. Copenhagen DR, Jahr CE (1989) Release of endogenous excitatory amino acids from turtle photoreceptors. Nature 341 (6242):536-539

126. Namiki S, Sakamoto H, Iinuma S, Iino M, Hirose K (2007) Optical glutamate sensor for spatiotemporal analysis of synaptic transmission. Eur J NeuroSci 25(8):2249-2259

127. Tsien RY (2005) Building and breeding molecules to spy on cells and tumors. FEBS Lett 579(4):927-932

128. Hires SA, Zhu Y, Tsien RY (2008) Optical measurement of synaptic glutamate spillover and reuptake by linker optimized glutamate-sensitive fluorescent reporters. Proc Natl Acad Sci U S A 105(11):4411-4416

129. Okumoto S, Looger LL, Micheva KD, Reimer RJ, Smith SJ, Frommer WB (2005) Detection of glutamate release from neurons by genetically encoded surface-displayed FRET nanosensors. Proc Natl Acad Sci U S A 102(24):8740-8745

130. Botvinick EL, Shah JV (2007) Laser-based measurements in cell biology. Methods Cell Biol 82:81-109

131. Luby-Phelps K, Taylor DL (1988) Subcellular compartmentalization by local differentiation of cytoplasmic structure. Cell Motil Cytoskeleton 10(1-2):28-37

132. Belaya K, Tollervey D, Kos M (2006) FLIPing heterokaryons to analyze nucleo-cytoplasmic shuttling of yeast proteins. RNA 12 (5):921-930

133. Patterson GH, Lippincott-Schwartz J (2002) A photoactivatable GFP for selective photolabeling of proteins and cells. Science 297(5588):1873-1877
134. Habuchi S, Ando R, Dedecker P, Verheijen W, Mizuno H, Miyawaki A, Hofkens J (2005) Reversible single-molecule photoswitching in the GFP-like fluorescent protein Dronpa. Proc Natl Acad Sci U S A 102(27):9511-9516

135. Ando R, Hama H, Yamamoto-Hino M, Mizuno H, Miyawaki A (2002) An optical marker based on the UV-induced green-to-red photoconversion of a fluorescent protein. Proc Natl Acad Sci U S A 99(20):12651-12656

136. Medina MA, Schwille P (2002) Fluorescence correlation spectroscopy for the detection and study of single molecules in biology. Bioessays 24(8):758-764

137. Manley S, Gillette JM, Patterson GH, Shroff H, Hess HF, Betzig E, Lippincott-Schwartz J (2008) High-density mapping of singlemolecule trajectories with photoactivated localization microscopy. Nat Methods 5(2):155-157

138. Betzig E, Patterson GH, Sougrat R, Lindwasser OW, Olenych S, Bonifacino JS, Davidson MW, LippincottSchwartz J, Hess HF (2006) Imaging intracellular fluorescent proteins at nanometer resolution. Science 313(5793): $1642-1645$

139. Shroff H, Galbraith CG, Galbraith JA, Betzig E (2008) Live-cell photoactivated localization microscopy of nanoscale adhesion dynamics. Nat Methods 5(5):417-423

140. Breusegem SY, Levi M, Barry NP (2006) Fluorescence correlation spectroscopy and fluorescence lifetime imaging microscopy. Nephron Exp Nephrol 103(2):e41-49

141. Petrasek Z, Krishnan M, Monch I, Schwille P (2007) Simultaneous two-photon fluorescence correlation spectroscopy and lifetime imaging of dye molecules in submicrometer fluidic structures. Microsc Res Tech 70(5):459-466

142. Petrasek Z, Schwille P (2008) Precise measurement of diffusion coefficients using scanning fluorescence correlation spectroscopy. Biophys J 94(4):1437-1448

143. Stiles JR, Bartol TM Jr (2001) Monte Carlo methods for simulating realistic synaptic microphysiology using MCell pp 87-127.

144. Stiles JR, Van Helden D, Bartol TM Jr, Salpeter EE, Salpeter MM (1996) Miniature endplate current rise times less than 100 microseconds from improved dual recordings can be modeled with passive acetylcholine diffusion from a synaptic vesicle. Proc Natl Acad Sci U S A 93(12):57475752 